\title{
LA-UR-21-21641
}

Approved for public release; distribution is unlimited.

Title:

Author(s):

Intended for: NEST College Course

NEST Lectures

Klumpp, John Allan
Issued:

2021-02-19 
Disclaimer:

Los Alamos National Laboratory, an affirmative action/equal opportunity employer, is operated by Triad National Security, LLC for the National Nuclear Security Administration of U.S. Department of Energy under contract 89233218CNA000001. By approving this article, the publisher recognizes that the U.S. Government retains nonexclusive, royalty-free license to publish or reproduce the published form of this contribution, or to allow others to do so, for U.S. Government purposes. Los Alamos National Laboratory requests that the publisher identify this article as work performed under the auspices of the U.S. Department of Energy. Los Alamos National Laboratory strongly supports academic freedom and a researcher's right to publish; as an institution, however, the Laboratory does not endorse the viewpoint of a publication or guarantee its technical correctness. 


\section{Interaction of Radiation with Matter}

Presented by John Klumpp, PhD, CHP

jaklumpp@lanl.gov 


\section{Homework}

- There will be homework ( $\mathrm{Hw}$ ) after each lecture

- One Hw will be worth 100 rem (100,000 mrem)

- Questions are worth a different number of mrem depending on difficulty

- We'll discuss answers in class the next day

- Hw 6 will be all extra credit 


\section{What is Radiation?}

- Photons (a.k.a. electromagnetic waves) and particles which have energy

- Ionizing radiation has enough energy to knock electrons out of atoms

- Cannot be seen or felt by humans, so we rely on special devices to detect it (e.g., Geiger counters) 


\section{Radiation Comes from Atoms}

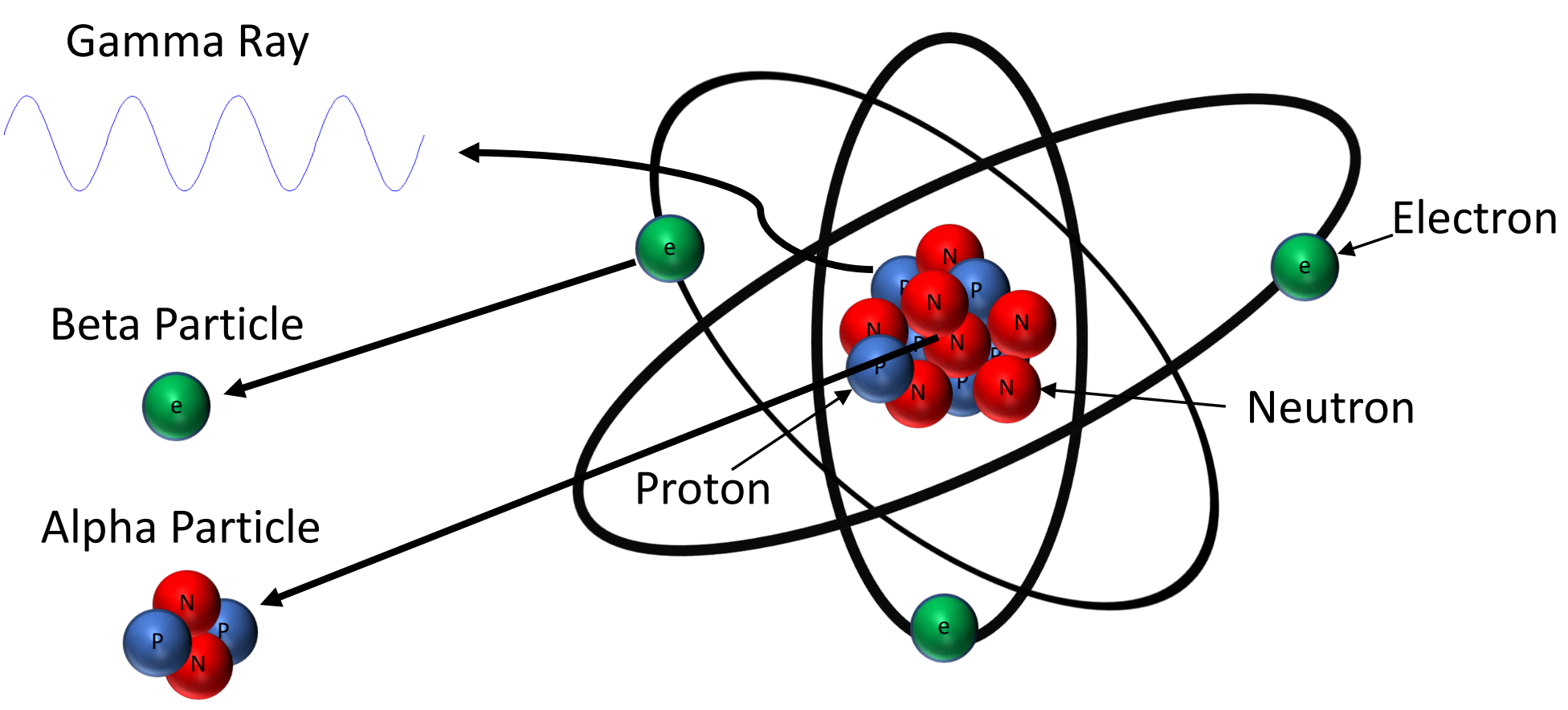

Atoms are composed of a nucleus of protons and neutrons orbited by electrons. Certain atomic nuclei spontaneously decay by emitting protons, neutrons, electrons, positrons, or photons. Photons ejected from atomic nuclei are called gamma rays, while ejected electrons are called beta particles. Some atoms will emit two protons and two neutrons stuck together - these are called alpha particles. 


\section{Isotopes}
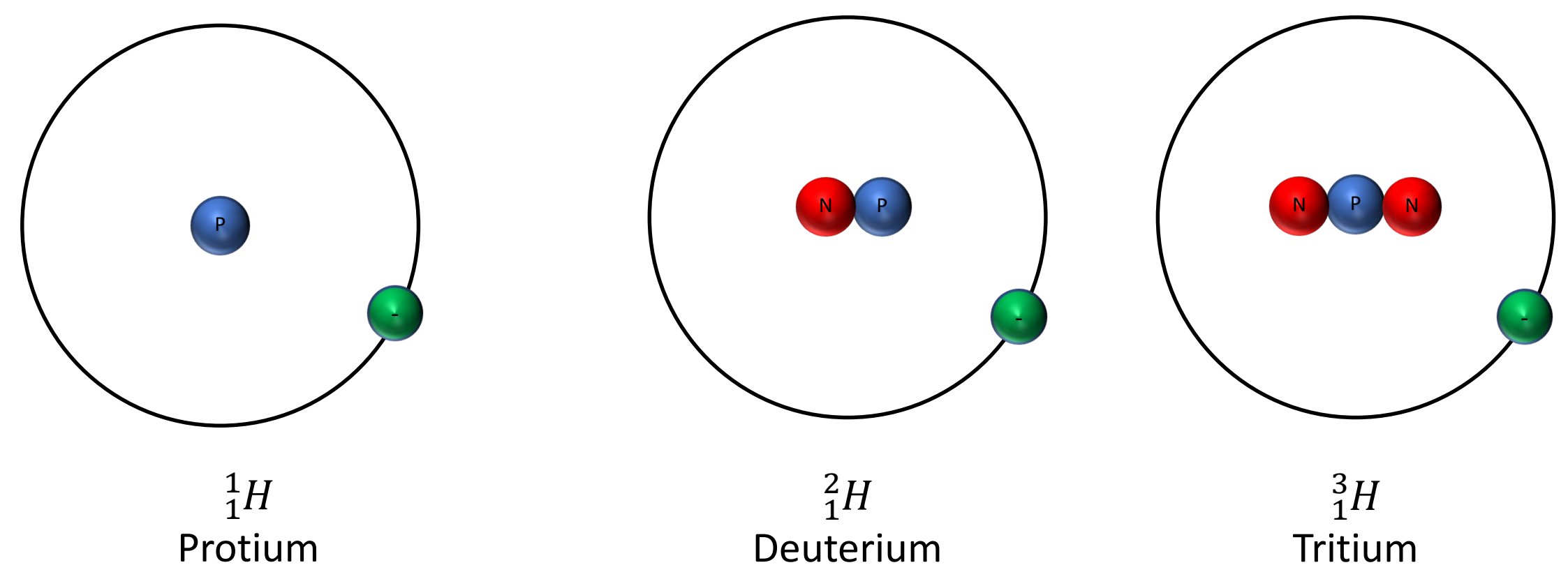

The isotopes of hydrogen are protium, deuterium, and tritium. They have zero, one, and two neutrons, respectively, but they all have one proton, meaning they are all hydrogen. $(Z=1, A=1,2,3)$ 


\section{Atoms}

- Building blocks of all matter

- Made of a shell of electrons surrounded by a dense nucleus of protons and neutrons

- Protons are charged - would fly apart if not held together by neutrons

- Some atomic nuclei are unstable and can spontaneously 'decay' 


\section{What are the different kinds of radiation?}

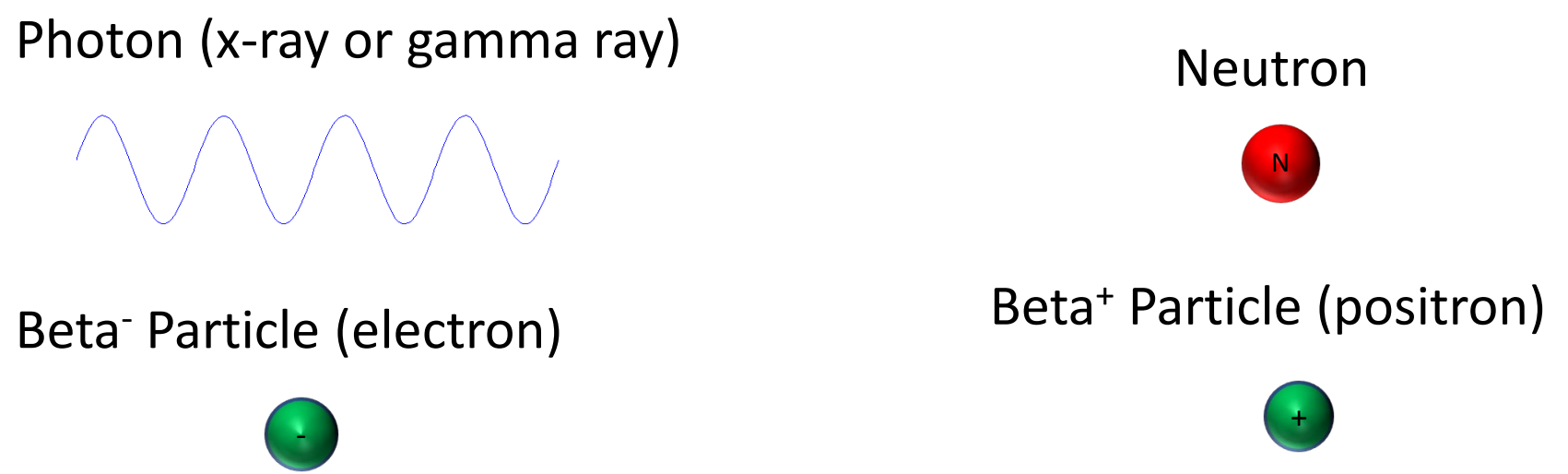

Alpha Particle

Alpha and beta particles have mass and an electric charge. Neutrons have mass but no charge. Photons are uncharged massless particles which travel at the speed of light. Unlike particles with mass, the amount of energy a photon has is determined by its wavelength. 


\section{Photons - Weightless, No Electric Charge}

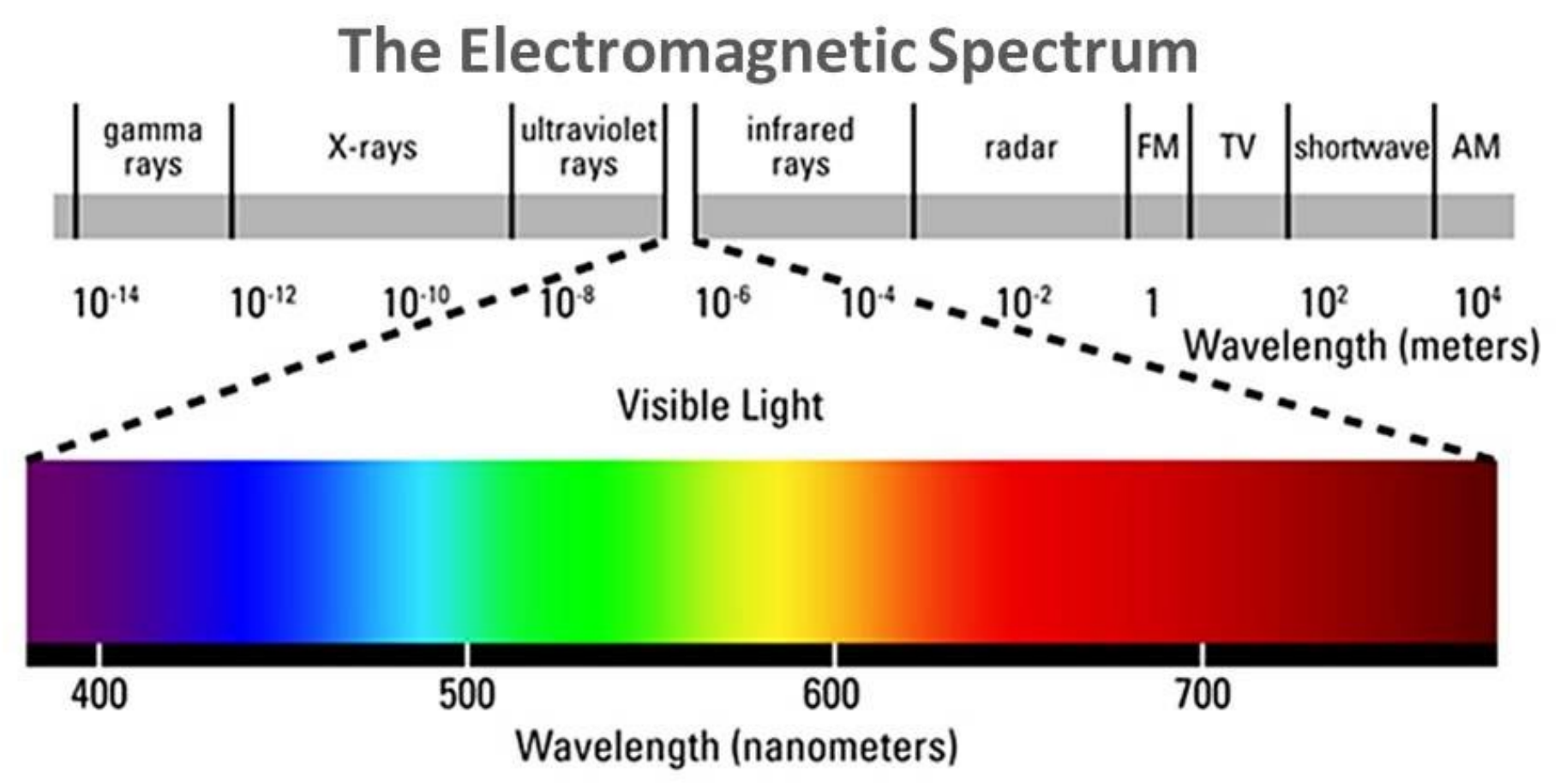

Photons are massless particles which travel at the speed of light. The amount of energy a photon has is determined by its wavelength. Photons with wavelengths less than about 10 nanometers (X-rays and gamma rays) have enough energy to ionize atoms, which makes them ionizing radiation. 


\section{Photons - Brown Bears and Grizzlies}

- Photons from decaying nuclei are called gamma rays, all other ionizing photons are called $\mathrm{x}$-rays

- Because they have no mass and no charge, high energy photons can only be blocked by several feet of dense material.

- Lower energy photons can be blocked by a thin lead apron

- Ultraviolet light is also ionizing. These photons are responsible for sunburns and increase the risk of skin cancer 


\section{Neutrons - Heavy, No Charge}

- No charge

- Mass: $1.67 \times 10^{-27} \mathrm{~kg}$ (thousands of times heaver than an electron)

- Mass: $9.11 \times 10^{-31} \mathrm{~kg}=0.511 \mathrm{MeV}-$ important because this is the photon energy when they annihilate

- Range $=0.5 \mathrm{~cm}$ per $\mathrm{MeV}$ in tissue, 12 feet per $\mathrm{MeV}$ in air

- Neutron $=$ Proton + electron + energy 


\section{Neutrons}

- Come from the nucleus of an atom; are relatively heavy, no charge.

- Come from decay of plutonium isotopes, nuclear fission and fusion

- Because they are not charged, they travel further through materials than electrons

- Are the only kind of radiation which can easily make other things radioactive 


\section{Beta Particles - Not Heavy, Charged}

- Mass: $9.11 \times 10^{-31} \mathrm{~kg}=0.511 \mathrm{MeV}$-important because this is the photon energy when they annihilate

- Electron Charge: $-1.6 \times 10^{-19} \mathrm{C}$

- Positrons: Same mass, positive charge, $1.6 \times 10^{-19} \mathrm{C}$

- Range $=0.5 \mathrm{~cm}$ per MeV in tissue, 12 feet per $\mathrm{MeV}$ in air 


\section{Beta Particles}

- High energy electrons or positrons (a.k.a. 'anti-electrons')

- Because of their charge, they can be blocked by a layer of clothing

- After an anti-electron runs out of energy, it annihilates with an electron and releases two high-energy $x$-rays

- Useful for cancer therapy and medical imaging 


\section{Alpha Particles - Heavy, Charged}

- Come from the nucleus of an atom; two protons, two neutrons stuck together (i.e., helium nuclei with no electrons)

- Weigh 8,000 times more than a beta particle and have double the charge

- Blocked by a sheet of paper, an inch of air, or the dead outer layer of a person's skin

- Only a hazard if taken into the body

- About 20x more effective at causing cancer 


\section{Where Does Radiation Come From?}

- Radioactive materials -e.g., Pu-239, Tc-99, K-40

- Particle Accelerators -e.g., x-ray machines, radiation therapy accelerators, LANSCE

- Nuclear Reactors

- Nuclear Weapons

- Stars 


\section{Decays and Counts}

- Radiation is formed when an atom decays - sometimes many particles/photons are released

- An atom can only decay once, because when it decays it turns into a new kind of atom (although the new kind of atom might also be radioactive)

- A large collection of atoms will result in a certain number of decays per second. Units: Becquerel $(\mathrm{Bq})=1$ decay/second. Curie $(\mathrm{Ci})=37$ billion decays/second.

- Usually a detector will not detect all of the radiation coming off a material. The detector will report counts per second (cps) 


\section{Interactions with Matter}

- Charged particles interact directly with electrons (they are called directly ionizing). So do photons.

- Uncharged particles (i.e., neutrons) interact with atomic nuclei (indirectly ionizing)

- Very energetic particles travel further between interactions (more penetrating)

- Heavier particles travel less far between interactions (less penetrating)

- Photon and neutron interactions are a bit more complicated 


\section{Interactions with Matter}

\section{LET AND DENSITY OF ENERGY LOSS EVENT}

a)

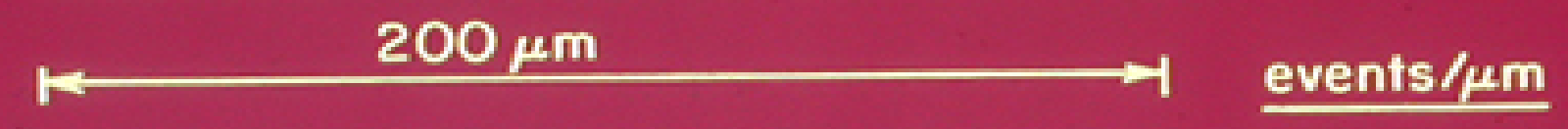

$\mathrm{Co}^{60}$

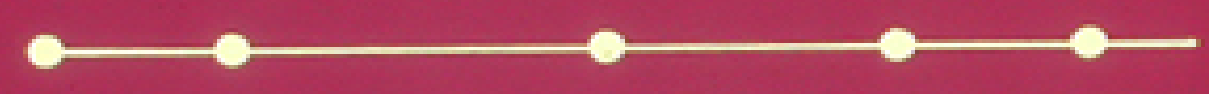

5

2 Mev proton

c)

5 Mev alpha

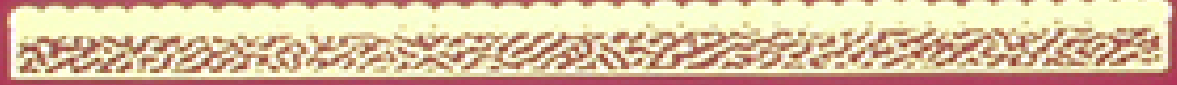

1500

b)

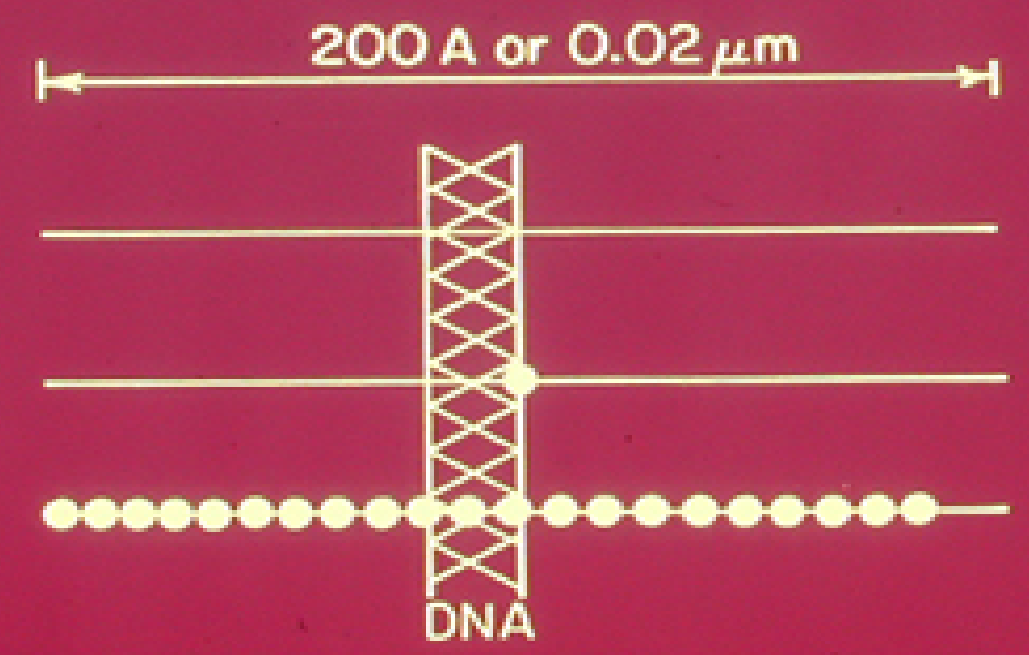

events/20A

$\mathrm{Co}^{60}$

2 Mev proton

5 Mev alpha 


\section{Interactions with Matter - Charged Particles}

- Charged particles interact directly with electrons (they are called directly ionizing). So do photons.

- Uncharged particles (i.e., neutrons) interact with atomic nuclei (indirectly ionizing)

- Almost all ionization is done by electrons

- Other radiation (i.e., photons, neutrons, alphas), give most of their energy to a relatively small number of electrons, which then do most of the ionization

- For example, when an alpha ionizes an atom, it gives the electron it kicked off enough energy to ionize many more atoms. It then travels some distance before ionizing another atom. 


\section{Interactions with Matter - Neutrons}

- Neutron interactions depend on speed

- "Fast" neutrons interact by bouncing off of nuclei like billiard balls - they loose the most energy bouncing off of things which weigh the same as them - i.e., hydrogen nuclei.

- "Slow" neutrons, aka thermal neutrons, get absorbed by nuclei - can sometimes make those nuclei radioactive

- Typical shielding is two layers - something with lots of hydrogen (e.g., water, plastic) to slow neutrons down, then something dense (e.g. cadmium) to absorb the slow neutrons. 


\section{Interactions with Matter - Photons}

- Photoelectric Effect:

-photon gives all of its energy to one electron

-Probability is proportional to $Z^{3} / \mathrm{E}^{3}$

-Not very probable over $1 \mathrm{MeV}$

- Compton Scattering:

-photon gives some of its energy to an electron

-Probability is proportional to Density/E (doesn't drop off as fast with energy)

-Most probable interaction between 0.5 and $5 \mathrm{MeV}$

- Pair production

-Photon interacts with atomic nucleus and produces electron/positron pair -Probability proportional to $\mathrm{EZ}^{2}$

-Only probable above $5 \mathrm{MeV}$ 


\section{Interactions with Matter - Absorbed Dose}

- Radiation absorbed dose is the amount of energy absorbed per kilogram of material ( $1 \mathrm{~Gy}=1 \mathrm{~J} / \mathrm{Kg})$

- Ionization takes energy - i.e., it takes some energy to knock electrons off of atoms.

- Energy absorbed is proportional to the number of ionized atoms

- Ionizations lead to chemical reactions which damage DNA, so absorbed dose should predict health effects

- But, it's more complicated than that... 


\section{Homework Questions}

1. Which is more harmful to cells - a $5 \mathrm{MeV}$ photon or a $5 \mathrm{MeV}$ alpha particle? Why?

2. You are measuring a material with $50 \mathrm{~Bq}$ (decays per second). Each decay causes one photon to be released. Your detector only detects $10 \%$ of the photons which are released. How many cps (counts per second) does your detector measure?

3. Can infrared radiation cause cancer? Why or why not?

4. Why is it best to use two different layers of shielding to block neutrons?

5. What is the difference between visible light and $\mathrm{x}$-rays? What is the difference between visible light and infrared radiation?

6. Pu-238 and Pu-239 are both of plutonium. 


\section{Biological and Health Effects of Radiation Exposure}

Presented by John Klumpp, PhD, CHP 


\section{Dose: How much is a Gray (Gy)?}

- Definition: $1 \mathrm{~Gy}=1 \mathrm{Joule} / \mathrm{kilogram}(\mathrm{J} / \mathrm{kg})$ - how much is that?

- Almost all the energy from radiation ends up as heat. How much?

- $4.186 \mathrm{~J}$ will heat up one gram of water by $1^{\circ} \mathrm{C}\left(1.8^{\circ} \mathrm{F}\right)$, so... $1 \mathrm{~J}$ will heat up a $\mathrm{kg}$ of water by $1 /(4186)^{\circ} \mathrm{C}=0.00024^{\circ} \mathrm{C}$

- You cannot feel radiation, even a lethal dose

- A note on units: $1 \mathrm{~Gy}=100$ rads $=100,000$ mrads 


\section{Dose: How much is a Gray (Gy)?}

- 1 Gy to your whole body all at once is enough to cause mild radiation sickness

- Definition: $1 \mathrm{~Gy}=1 \mathrm{Joule} / \mathrm{kilogram}(\mathrm{J} / \mathrm{kg})$ - how much is that?

- How many $x$-rays for one Joule? ${ }^{60}$ Co gamma rays are about $1 \mathrm{MeV}$. In joules, this is $1.6 \mathrm{x}$ $10^{-13} \mathrm{~J}$ (charge of an electron multiplied by one volt).

So, $1 \mathrm{~J}=1 /\left(1.6 \times 10^{-13}\right)=6,250,000,000,000$ photons

- Typical person weighs about $60 \mathrm{~kg}$, so multiply the number above by 60 : to get one Gy from ${ }^{60} \mathrm{Co}$, a person would need to absorb about $375,000,000,000,000$ ( 375 trillion) gamma rays.

- This is why you shouldn't freak out when you hear one click on a Geiger counter

- But a Gray is hardly enough to make you sick. What happens to all those gamma rays? 


\section{Health Effects Come from Damaged DNA}

- Radiation ionizes molecules in cells

- Sometimes this directly damages DNA

- More often it leads to a series of chemical reactions which eventually damage DNA

- Examples of free radicals: $\mathrm{O}_{2}{ }^{-}, \mathrm{OH}^{-}, \mathrm{H}^{+}$

- $\mathrm{OH}^{-}$reacts near where it is formed, $\mathrm{O}_{2}{ }^{-}$can diffuse long distances

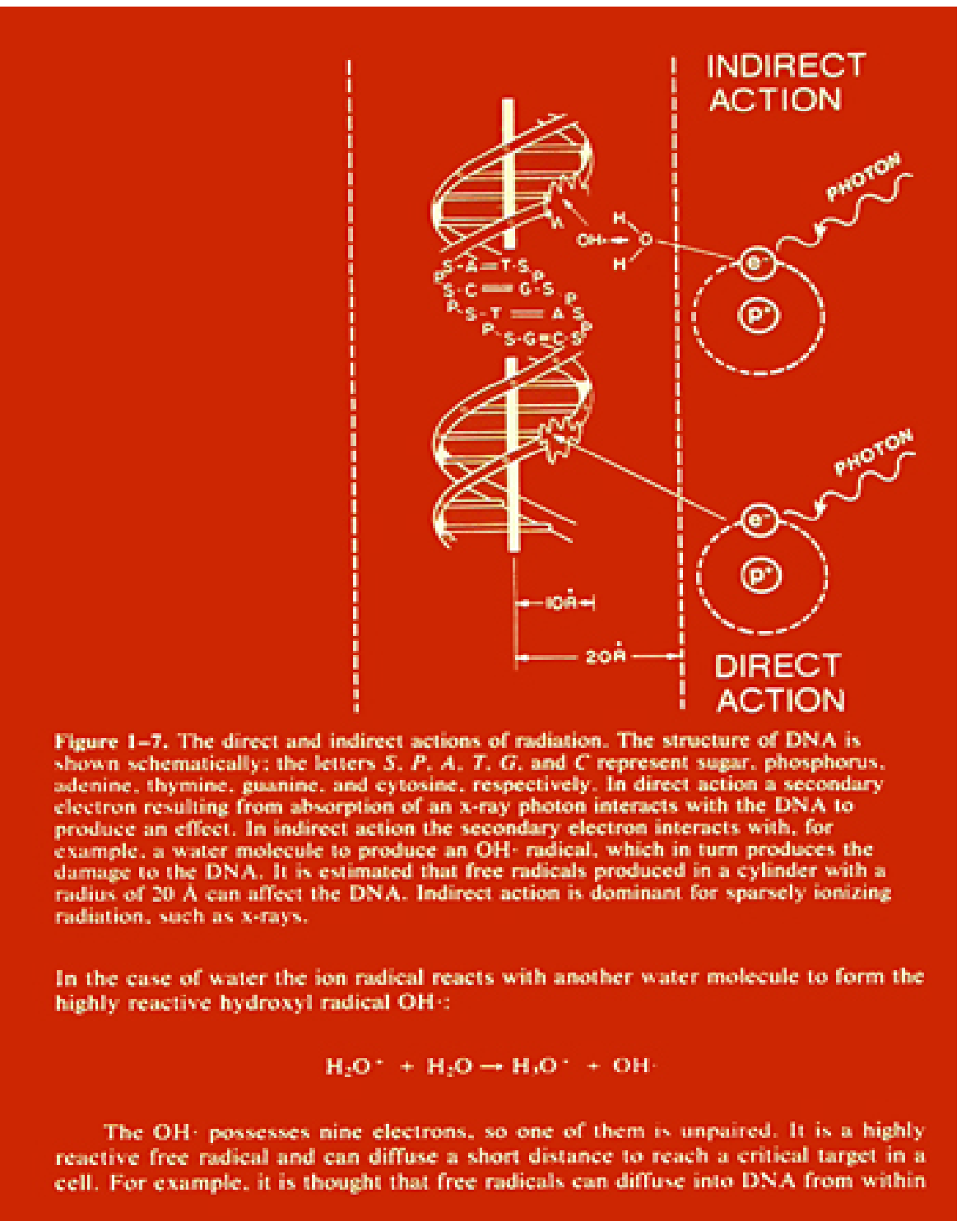




\section{What Happens Next?}

- Radicals can be 'repaired' i.e., neutralized before causing harm by a number of chemicals and enzymes which occur naturally (e.g., antioxidants) or which your body makes(e.g., peroxidase)

- Some damage other parts of the cell (typically not enough to cause harm), but a few damage DNA

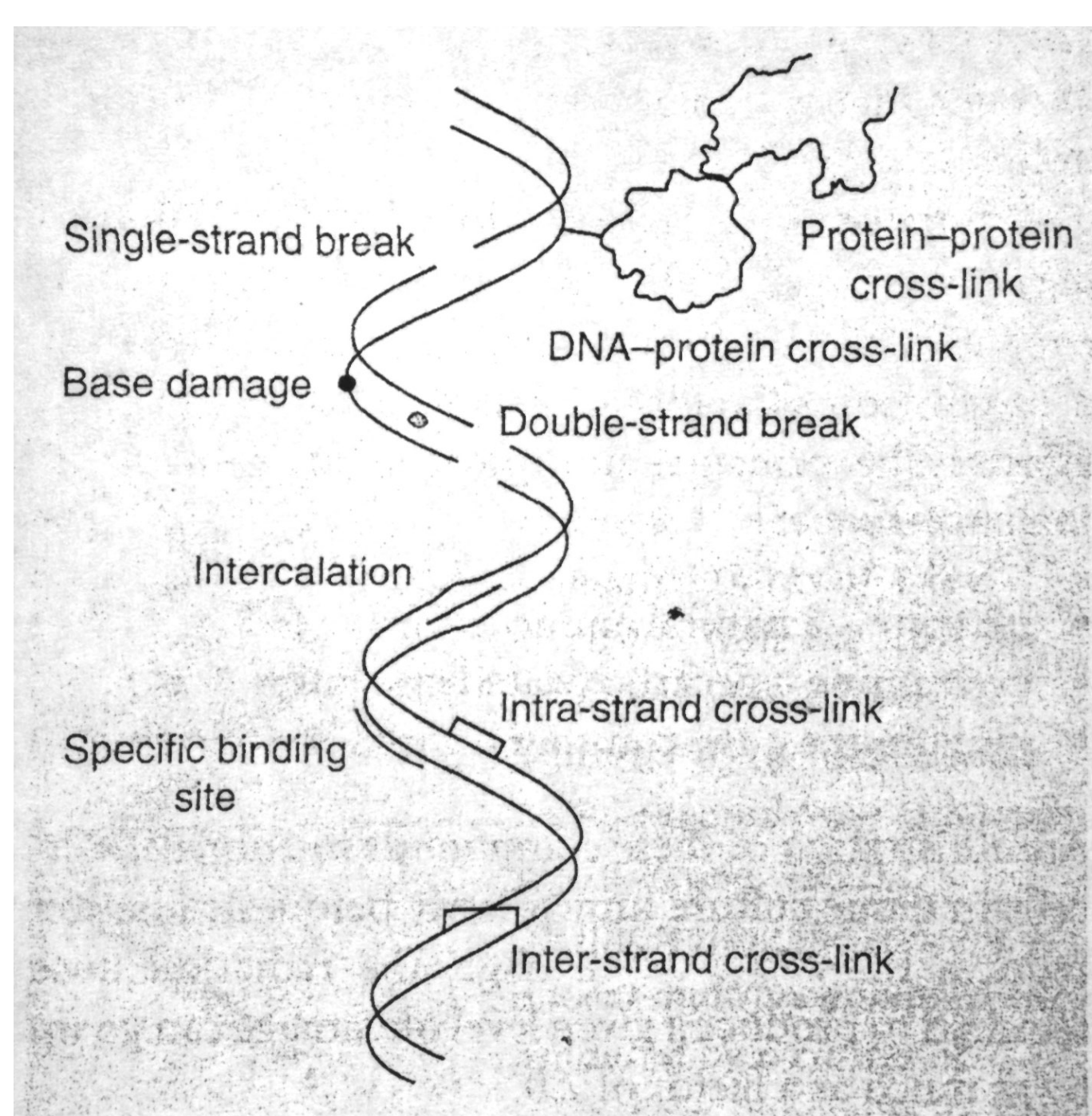

Figure 8.3 Types of damage to DNA produced by radiation and chemical agents. 


\section{Damaged DNA is Not Usually a Problem}

- DNA is damaged all the time, and sometimes errors occur when cells replicate - the body has evolved several ways to deal with this

- Repair: DNA contains two strands which complement each other - if one strand is broken, the other strand serves as a backup, and is used to repair it - this is the most common outcome of DNA damage

-What if both strands get broken? Or what if the body can't tell if there was damage? 


\section{Unrepairable DNA Damage}

- The body has several ways to deal with unrepairable damaged DNA

- Often the damage kills the cell, or prevents it from replicating (usually no problem)

- Other times the cell 'knows' that it is damaged and self destructs - damaged cells may also send out signals instructing nearby cells to self-destruct as well.

- Other times immune cells can tell that the cell is damaged and destroy it

- Sometimes none of these things happen, and the cell replicates, passing on the mutation; in these cases, the mutation is usually harmless, but not always. 


\section{Examples of Faulty Repair (read top to bottom)}

A
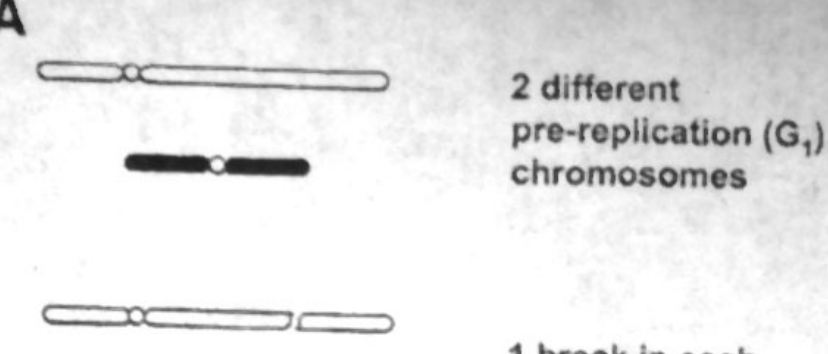

ח

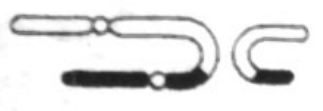

Illegitimate unions

Replication (S)

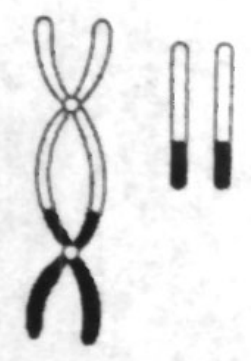

1 break in each chromosome
B

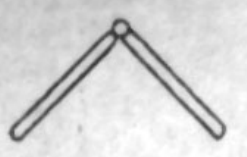

Pre-replication

(G) chromosome
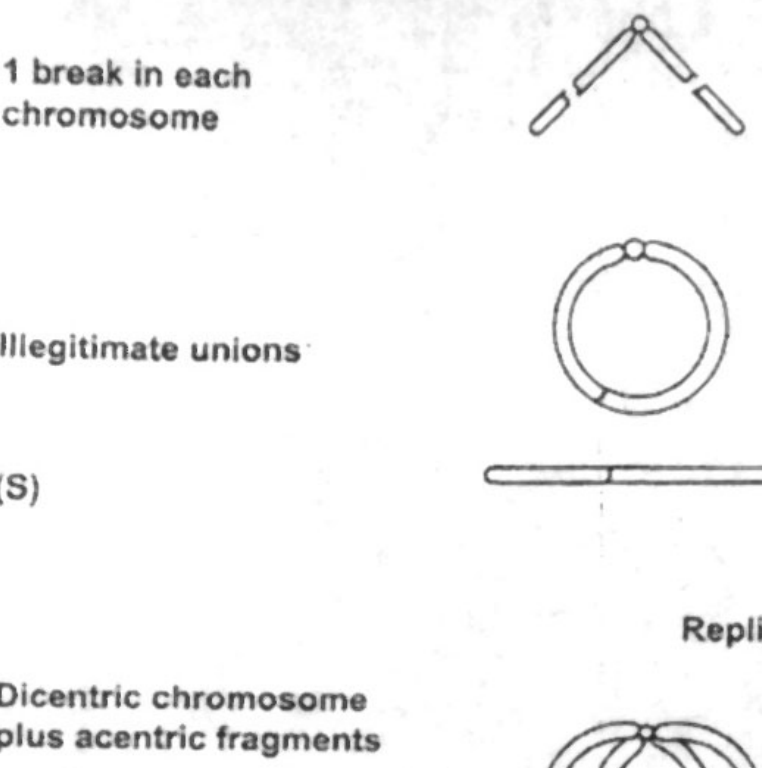

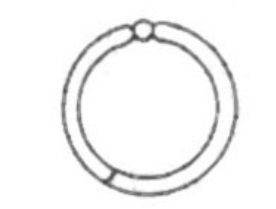

Incorrect unions

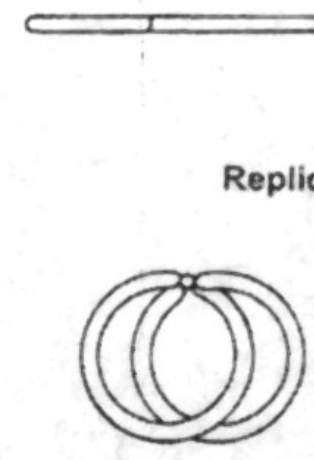

Replication (S)

Overlapping rings

Acentric fragments
C

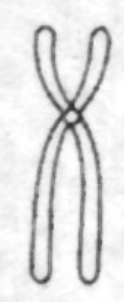

Post-replication $\left(\mathrm{G}_{2}\right)$

chromosome

\section{Breaks in both arms of} the same chromosome

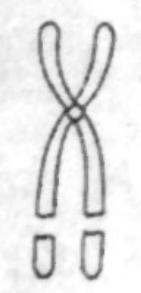

Break in each

chromatid

(isochromatid deletion)

00

Sister unions

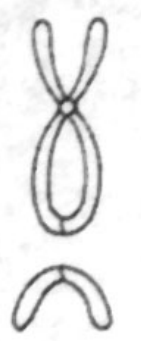

Dicentric chromatid, plus acentric chromatid fragment 


\section{Broken Chromosomes}

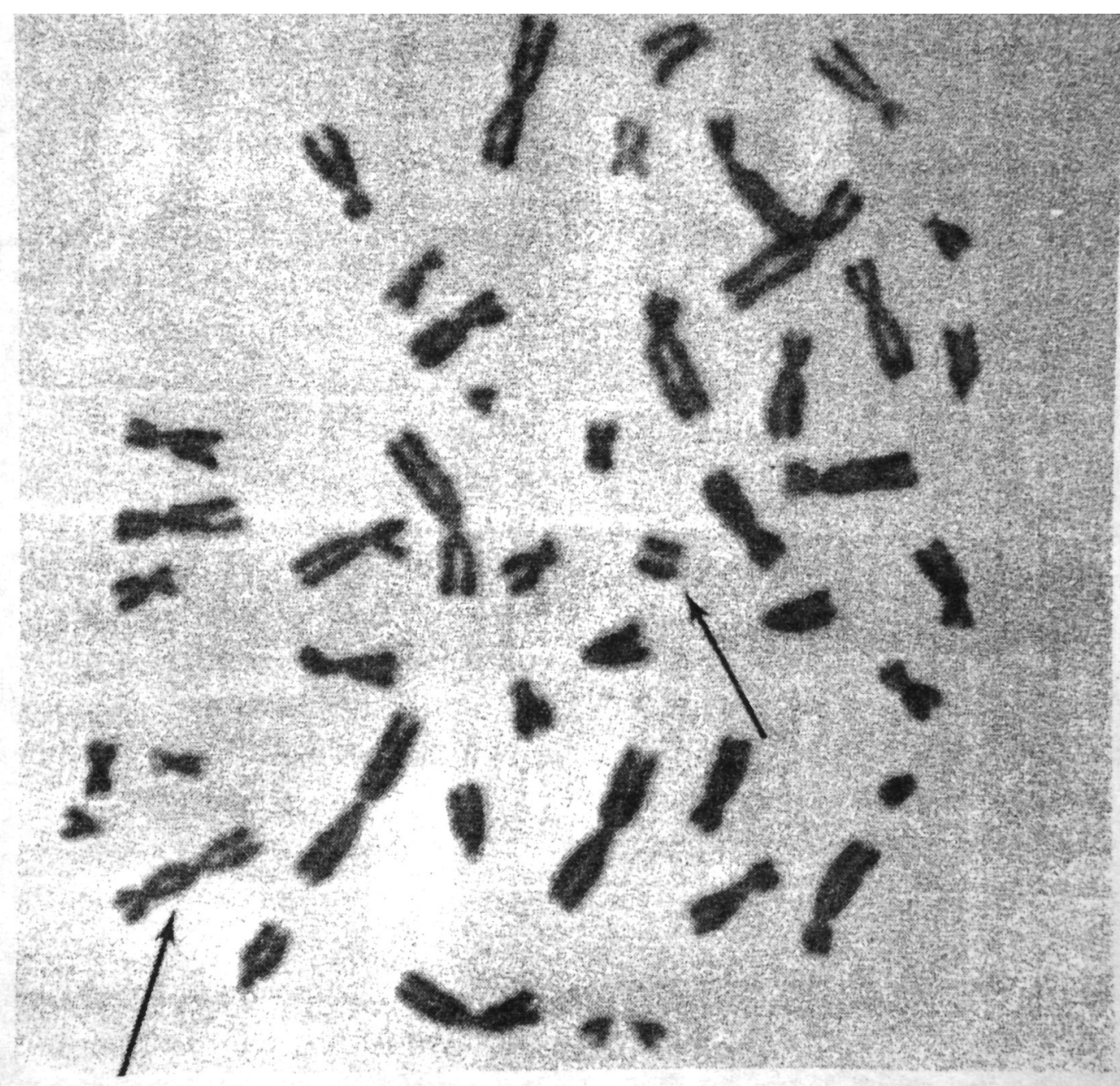

FIGURE 2.7 Radiation-induced chromosome aberrations in human leukocytes viewed at metaphase. A: Normal metaphase. B: Dicentric and fraament (arrows). (Continued) 


\section{Radioprotecting Agents}

- Some drugs exist which can prevent radiation sickness if given immediately before or after a large exposure

- They make you feel very sick, only used for huge doses

- But there may be other options...

J. RADIAT. RES., 43, 237-245 (2002)

Drinking Beer Reduces Radiation-induced Chromosome Aberrations in Human Lymphocytes

MANAMI MONOBE ${ }^{1,2}$ and KOICHI ANDO ${ }^{2 *}$

Beer / Radioprotection / Chromosome aberration / X rays / Heavy ions

We here investigated and reported the effects of beer drinking on radiation-induced chro-

mosome aberrations in blood lymphocytes. Human blood that was collected either before or after drinking a $700 \mathrm{ml}$ beer was in vitro irradiated with $200 \mathrm{kVp} \mathrm{X}$ rays or $50 \mathrm{keV} / \mu \mathrm{m}$ carbon ions. The relation between the radiation dose and the aberration frequencies (fragments and dicentrics) was significantly $(\mathrm{p}<0.05)$ lower for lymphocytes collected $3 \mathrm{~h}$ after beer drinking than those before drinking. Fitting the dose response to a linear quadratic model showed that the alpha term of carbon ions was significantly $(\mathrm{p}<0.05)$ decreased by beer drinking. A decrease of dicentric formation was detected as early as $0.5 \mathrm{~h}$ after beer drinking, and lasted not shorter

than $4.5 \mathrm{~h}$. The mitotic index of lymphocytes was higher after beer drinking than before, indi- 


\section{Different Kinds of Dose}

- Absorbed dose, discussed last week: Defined as radiation energy absorbed per unit mass of tissue

-Units: $1 \mathrm{~Gy}=100$ rads $=100,000$ mrads

-Good for predicting immediate health effects

-Less good for predicting cancer

-A physical (measured) quantity - no calculations needed

- Equivalent dose: Dose to an organ or tissue scaled by how effective the radiation is at causing cancer

-Units: $1 \mathrm{~Sv}=100 \mathrm{rem}=100,000$ mrem

$-1 \mathrm{~Gy}$ from $\mathrm{x}$-rays = $1 \mathrm{~Sv} ; 1 \mathrm{~Gy}$ from alphas $=20 \mathrm{~Sv}$

-Usually given for a particular organ rather than whole body

-Misused as limit against immediate health effects (e.g., limit to heart is

50 rem)

-Confusing, not very useful 


\section{Different Kinds of Dose}

- Effective Dose (ED): Sum of equivalent doses to organs and tissued scaled by how likely they are to develop cancer

-Units: 1 Sv $=100$ rem $=100,000$ mrem

-Risk of cancer from $1 \mathrm{~Gy}$ to whole body from $\mathrm{x}$-rays equals risk of cancer from $1 \mathrm{~Sv}$ from any radiation exposure to any part of the body

$-1 \mathrm{~Sv}(100 \mathrm{rem})=5 \%$ risk of developing cancer

- 50-year Committed Effective Dose - a.k.a. CED: Effective dose that will occur because of a radionuclide intake for the 50 years after intake happens

-Units: $1 \mathrm{~Sv}=100 \mathrm{rem}=100,000$ mrem

-If you inhale something radioactive in 2021, and it gives you 3 rem in 2021, 2 rem in 2022, 1 rem in 2023, and then no more, the CED(50) for 2021 is 6 rem

-Assumed that cancer risk from 1 Sv CED $=5 \%$ (probably isn't)

-Regulatory limit to radiation workers for ED+CED: 0.05 Sv (5 rem), so in the example above the worker exceeded the regulatory limit 


\section{Effects of Radiation}

- Deterministic Effects

-Always happen above some dose threshold, but never below the threshold -Effects are worse beyond the threshold -Examples: radiation burns, radiation sickness, birth defects

-Lowest threshold is about 25 - 50 rads, which causes a detectable drop in number of blood cells (not enough to cause symptoms)

- Stochastic effects

-i.e., an effect on your chance of getting cancer

-Because effect is random, can only be observed in populations of people -Increased chances of getting cancer observed for doses larger than about 10 rem -For protection purposes, assumed for all radiation exposures (no threshold). It is hotly debated whether smaller doses really do increase risk of cancer 


\section{Background Radiation}

- Common reason for false alarms, and a useful reference when asking, "should I be worried?"

- Dose from background varies depending on geology and elevation:

-Florida: $0.131 \mathrm{rem} /$ year (50 year CED $=6.55 \mathrm{rem}$ )

-South Dakota: $0.962 \mathrm{rem} /$ year ( 50 year $\mathrm{CED}=48.1 \mathrm{mrem}$ )

-Los Alamos: $0.520 \mathrm{rem} /$ year (50 year CED $=26 \mathrm{rem}$ )

- Most natural background is radon inhalation (like plutonium, an internal alpha emitter)
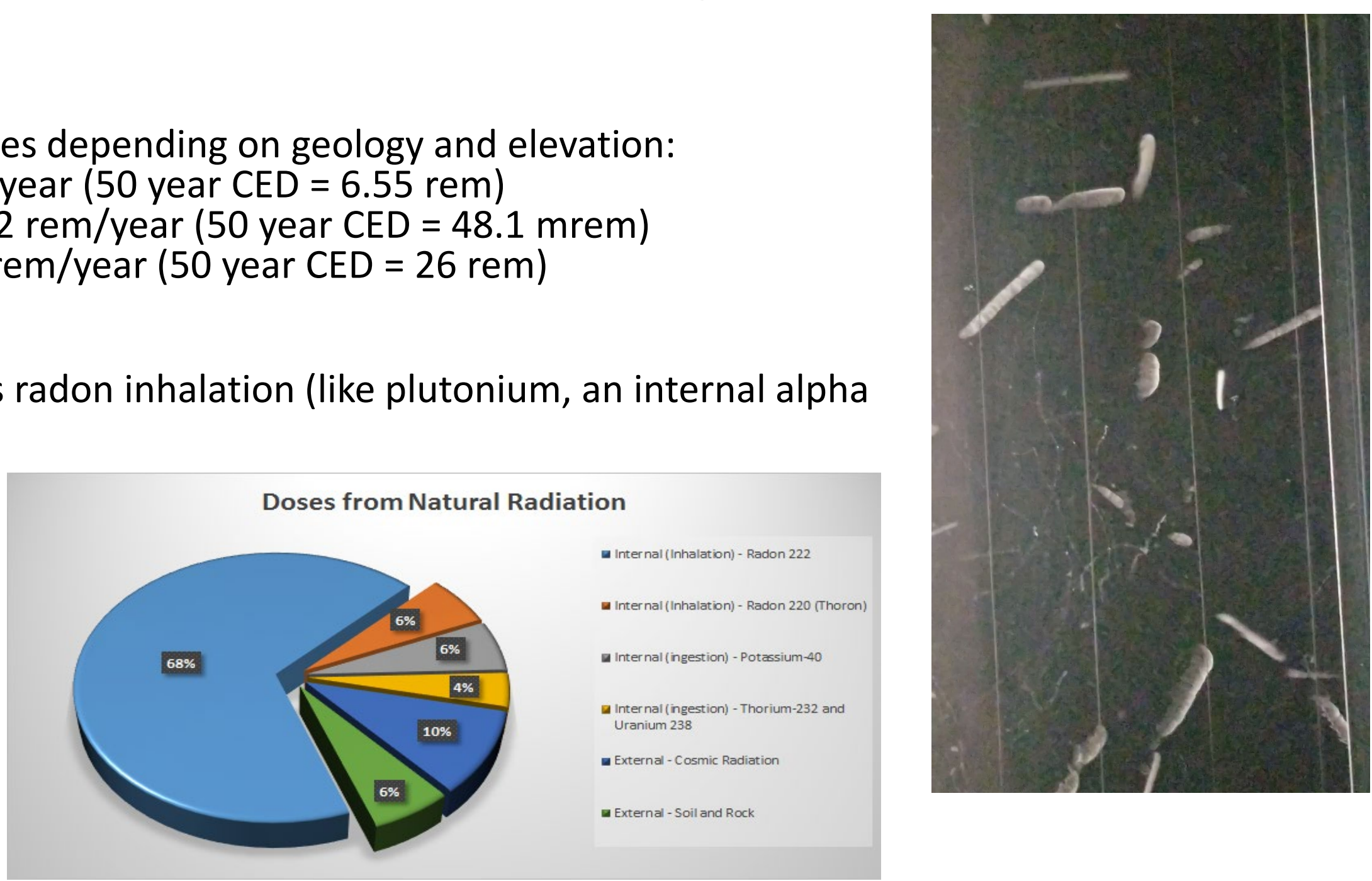


\section{Stochastic Effects}

- Effective dose (Sv/rem) is used to estimate risk of fatal cancer

- 1 Sv (100 rem) increases the risk of dying from cancer by about $5.5 \%$

- Effect is linear, so $0.1 \mathrm{~Sv}$ (10 rem) increases risk by about $0.55 \%$

- About $40 \%$ of all Americans will get cancer at some point in their lives, and half of those ( $20 \%$ of all Americans) will die from cancer

- Small changes in risk from small doses are very difficult to detect - no one has every detected a change in cancer risk from doses smaller than $0.1 \mathrm{~Sv}$

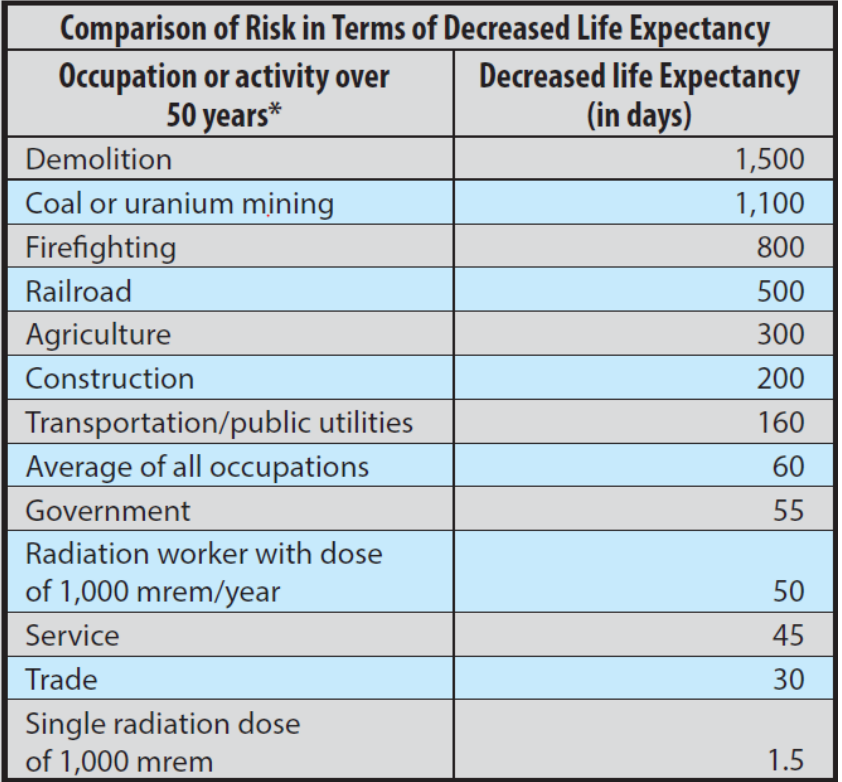




\section{Acute (Immediate) Health Effects}

- Absorbed dose is good for predicting immediate health effects

- Large doses (>0.5 Gy) usually needed to cause observable acute effects

- Radiation sickness results from radiation of bone marrow, intestines, and brain (typically whole-body radiation)

- Irradiation to specific tissues only damages those tissues (e.g., skin burns)

- The body is very good at repairing itself, so dose rate is important 


\section{Acute Health Effects Depend on the Dose, Dose Rate, How Much of Body Gets Irradiated}

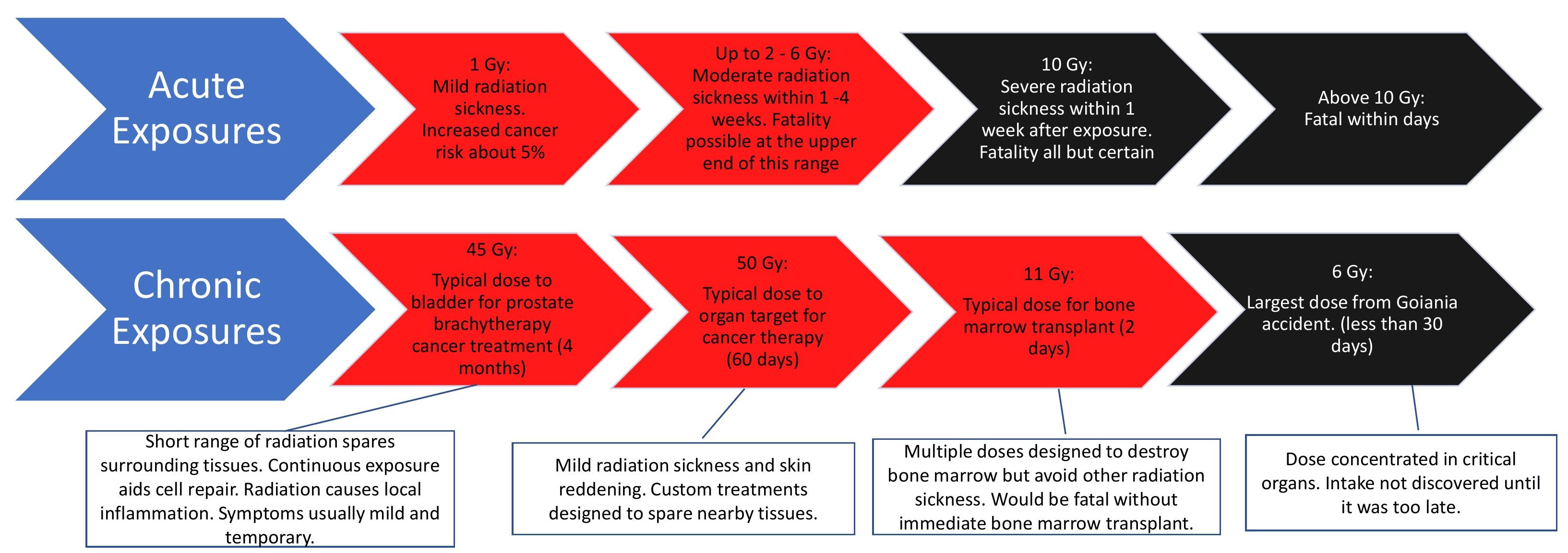

\section{reddening. Custom treatments} designed to spare nearby tissues.
Dose concentrated in critical it was too late. 


\section{Radiation Sickness}

- Happens when you get more than about $1 \mathrm{~Gy}$ to the whole body all at once

- Three Phases

-Prodromal Phase

-Latent period

-Hematopoeietic, Gastrointestinal, or Central Nervous System Syndromes

- Severity, type of syndrome, and outcome all depend on dose 


\section{Prodromal Phase}

- Happens within 24 to 36 hours after exposure. Larger dose: sooner and more severe

- Loss of appetite, nausea (survival probable), vomiting (survival questionable), diarrhea (survival unlikely if happens in first 12 hours)

- Person starts feeling better soon

- Not well understood - we don't really know why it happens 


\section{Latent Period}

- Early symptoms go away and the person starts to feel better

- The smaller the dose, the longer the latent period (and vice versa)

- A small dose can give up to a 3 week latent period; very large doses (>10 Gy) can give little or no latent period) 


\section{Hematopoetic/Bone Marrow Syndrome}

- Happens for doses greater than 1-2 Gy

- Reduces the stem cells in bone marrow which make your blood cells

- Latent period of $2-3$ weeks

- Sickness onset when the existing blood cells reach the end of their natural lifespans and are not replaced

- Symptoms: bleeding, bruising, vulnerability to infection

- Depending on dose, usually survivable with proper medical care 


\section{Leukocyte (white blood cell) count based on dose to Chernobyl patients}

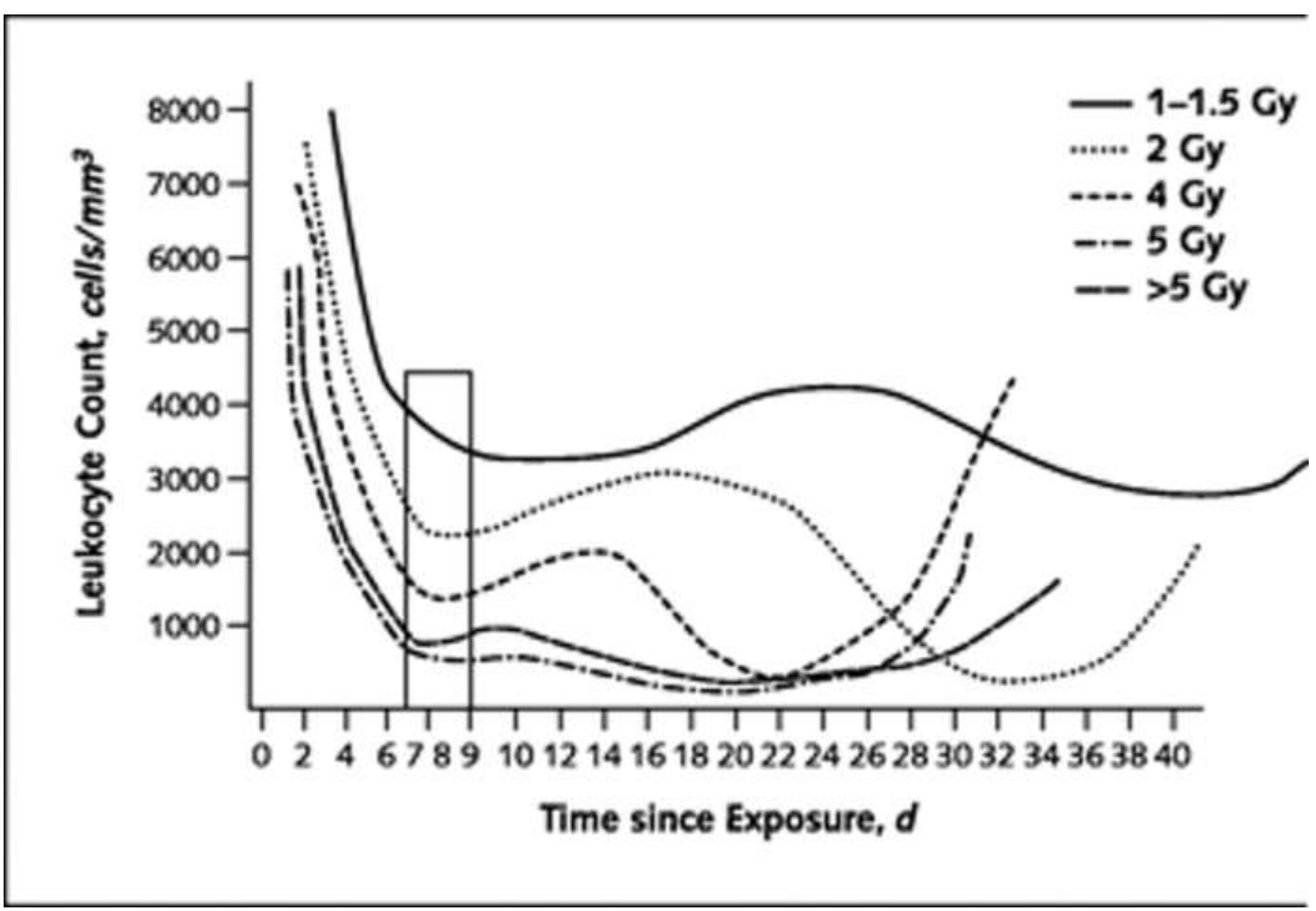




\section{Gastrointestinal (GI) Syndrome}

- Happens for doses greater than about $5 \mathrm{~Gy}$

- Reduces stem cells which replace cells lining the intestines

- Diarrhea in the prodromal phase

- 4- 5 day latent period

- Patient will also develop hematopoetic syndrome

- The higher the dose, the higher chance of death. May be survivable with intense medical care if dose is under about $10 \mathrm{~Gy}$. 


\section{Central Nervous System (CNS) Syndrome}

- Happens for doses greater than $20 \mathrm{~Gy}$

- Brain must be irradiated to cause CNS Syndrome

- No latent period

- Patient is immediately confused and dizzy, rapidly falls into a coma

- 50 Gy to the brain causes death in $2-3$ days 


\section{Radiation Exposures During Pregnancy}

- Quite a bit is known from following atomic bomb survivors

- Risks depend on dose and timing - No health effects observed below $0.1 \mathrm{~Gy}$ (10 rads)

- Fetal development can be impacted during weeks $2-24: 0.1-0.5 \mathrm{~Gy}$ ( $10-50$ rads) increases chance of low birth weight. Above $0.5 \mathrm{~Gy}$ (50 rads) the chances of miscarriage and birth defects increase

- After 24 weeks, essentially no risk for birth defects

- Large doses to the gonads (large enough to cause radiation sickness) may induce temporary sterility for several weeks or months in men and women. But no increased risk of birth defects, cancer, or miscarriage with subsequent children. 


\section{Radiation Exposures on Fertility}

- Large doses to the gonads (usually associated with cancer radiation therapy) can cause temporary or permanent infertility

- In males, 15 rads can cause temporary sterility (lasting weeks to months). Permanent sterility occurs in doses greater than $6 \mathrm{~Gy}$.

- In females, infertility can happen with doses around 4-6 Gy.

- If infertility doesn't happen, or if it is temporary, future offspring are healthy 


\section{References}

Hall, E; Giaccia, A. Radiobiology for Radiobiologist. Walters Kluwer. June 2018.

Steel, G. Basic Clinical Radiobiology, $3^{\text {rd }}$ Ed. CRC Press. July 2002.

Cember, H. Johnson, T. Introduction to Health Physics. McGraw-Hill. August 2008. 


\section{Homework Questions}

- A friend of yours gets a report that they received 500 mrem from radiation at work. They tell you that they are starting to feel nauseous and think they have radiation sickness. What would you tell them?

- How can radiation damage cells even when it doesn't hit the DNA?

- Suppose radiation damages some DNA in a cell. Will the cell most likely: a) repair itself, b) die, or c) become cancerous?

- A friend of yours is receiving radiation therapy for cancer. As part of their treatment, they receive 50 Gy over two months. Why doesn't your friend die of radiation sickness?

- You are involved in a major radiation accident at work, but the exact dose is unkown. You feel fine until the next day when, about 36 hours after the accident, you start to feel sick and lose your appetite. Before long you feel better, but three weeks later you start feeling sick again. What is wrong with you? Are you likely to be OK? Estimate your dose.

- You are involved in a radiation accident while 5 months pregnant. Your estimated dose is 7 rem, which is higher than the regulatory limit for radiation workers. What is likely to happen to the baby?

- Why is inhaling radon similar to inhaling plutonium?

- Suppose you decide to spend the summer (3 months) with family in North Dakota instead of in Los Alamos. How much higher is your radiation dose for the year compared to what it would be if you had stayed in Los Alamos. 
Radionuclide Detection and Dose Assessment

Presented by John Klumpp, PhD, CHP 


\section{Detectors and Dosimeters}

- Used to detect, identify radionuclides

- Many different kinds, tradeoffs

-Some are good for specific types of radiation like photons

-Some are portable and some are not

-Some do spectroscopy (identify radiation energy), some do not

-Some are very sensitive, some are better for high-radiation fields

-Some give immediate results (e.g., audible clicks), some do not

- Often used to find contamination or alert workers to potential danger

- Dosimeters used to estimate doses to individuals 


\section{Gas Detectors}

- Put gas (e.g., air) between two electrodes

- If an ionization happens, the free electron will fly towards the positively charged electrode, and the positively charged nucleus will go towards the negatively charged electrode.

- This creates a current, which can be detected

- Many different types 


\section{GM (Geiger) Counters}

- So much voltage that the ionized atom ionizes more gas as they fly towards the electrodes

- Current is always the same no matter what kind of radiation entered

- Very sensitive - can detect a single particle or photon

- Cannot tell you about dose

- Need some time to 'recover' after a detection - not good for high count rates 


\section{Proportional Counters}

- Current is proportional to the voltage between electrodes

- Alphas and betas produce different currents

- Can be used to distinguish between different types of radiation

- Sometimes useful for surveying if looking for a particular thing 


\section{Ionization Chambers}

- Produce a different current depending on how much 'dose' the detector received

- Can give dose estimates

- Accurate dose estimates only for a range of photon energies - not good for very energetic photons; can be used for betas but it's tricky

- Not very sensitive - not great for surveying for contamination 


\section{Gas Detectors}

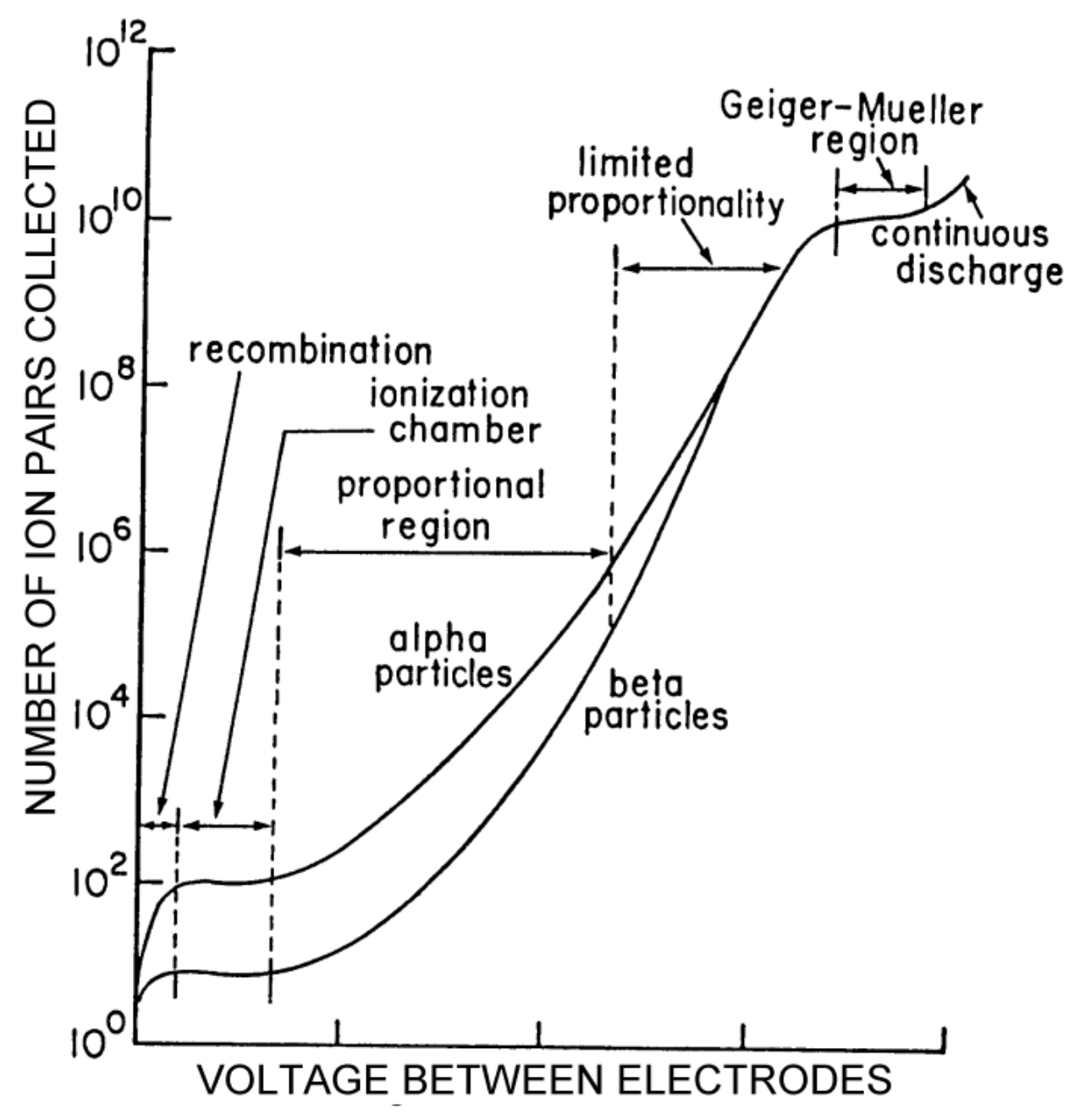




\section{Semiconductor Detectors - HPGe, GeLi, SiLi}

- "Solid state," but same idea - incoming radiation knocks loose an electron, induces a current

- HPGe has very high resolution for photon energy - used to identify unknown radionuclides

- Can be calibrated to give dose rate estimates

- Not typically very sensitive - not great for monitoring for contamination

- Sometimes used for 'real-time' personnel monitoring (wear it on your belt and it will alarm if the dose rate goes up. 


\section{Scintillation Detectors}

- Made of clear crystals which emit a flash of light when radiation hits them

- Higher energy photons $\rightarrow$ brighter light, so you can learn about the energy

- All have a photomultiplier tube (PMT) which amplifies the flash, and a special material which turns the light into electricity

- Common scintillators: Nal, ZnS, LSC fluid 


\section{Gamma Spectroscopy}

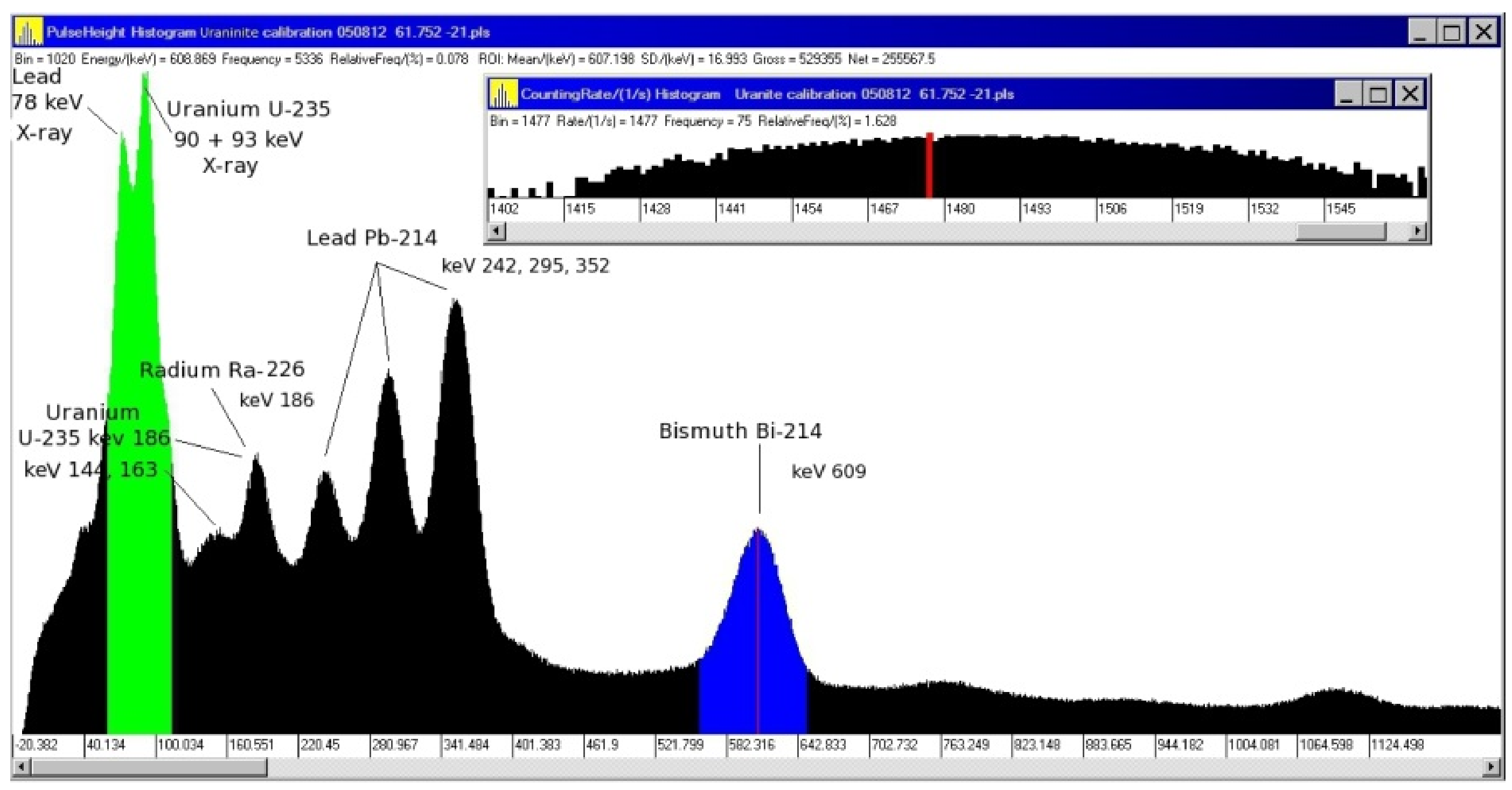




\section{Solid-State Scintillators}

- Solids are much more dense than gas (more atoms for radiation to crash into), so more sensitive to gammas than gas detectors

- Nal is the most commonly used for gamma detection - not as high resolution as HPGe, but much more sensitive, inexpensive, and portable

- ZnS is used in portable alpha detectors - you can find them at LANL Pu facilities 


\section{Alpha Spectroscopy}

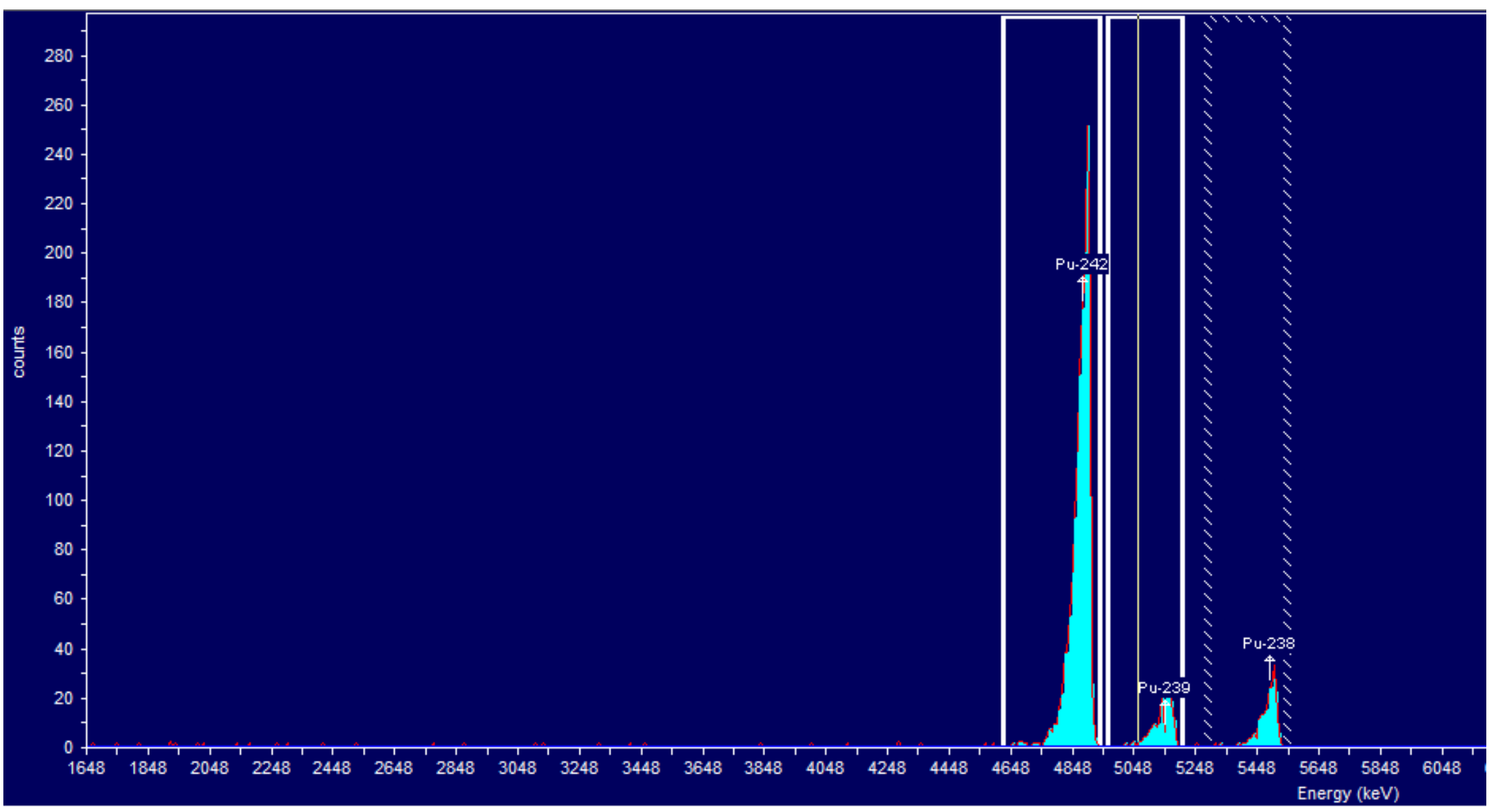

Alpha spectroscopy measures the energy of the alpha which are emitted when radioisotopes like Plutonium-239 decay. Different isotopes emit alpha particles with different amounts of energy. This allows us to measure the amount of each isotope in the sample. 


\section{Liquid Scintillation}

- Liquid scintillation is commonly used for alphas and low-energy betas: e.g., from tritium $\left({ }^{3} \mathrm{H}\right),{ }^{14} \mathrm{C}$

- How to get material into the liquid? Material is usually collected on a swab. Chemical separation might be needed.

- Also used for tritium bioassay

- Extremely sensitive, but not portable 


\section{Liquid Scintillation}

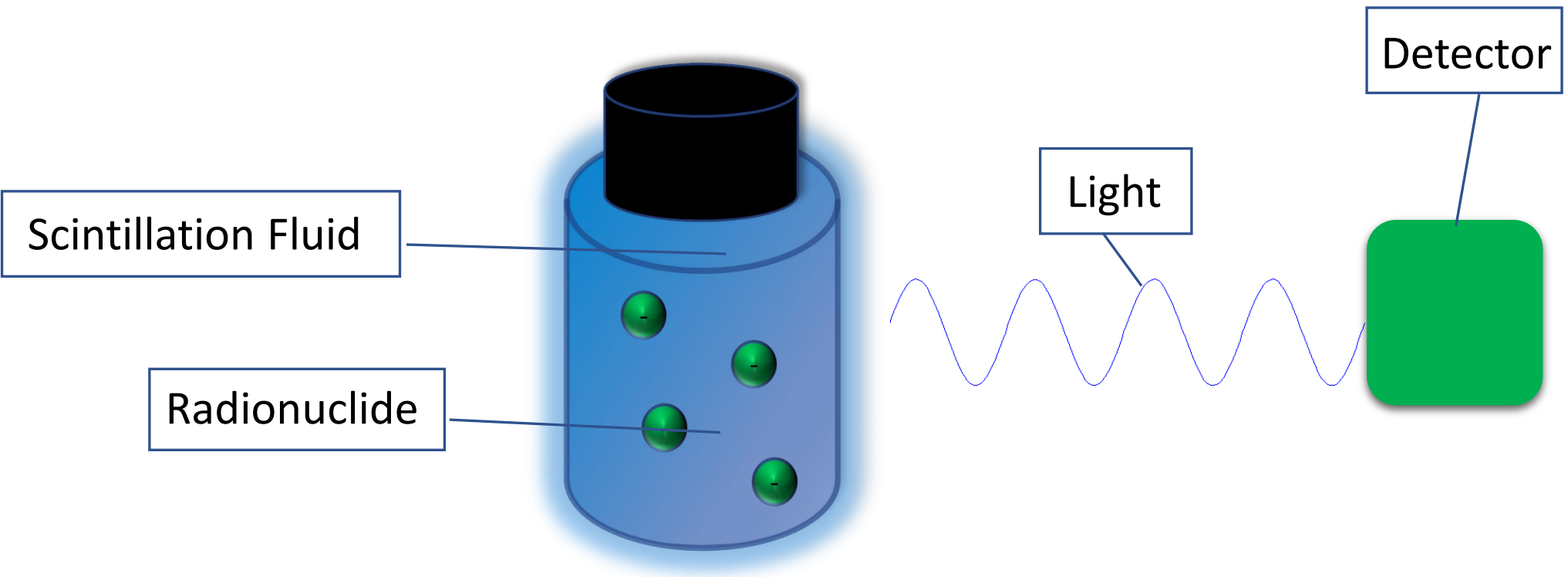

Samples are mixed with a clear liquid which emits light (i.e., scintillates) whenever it absorbs radiation. The amount of light emitted by the liquid tells us how much radiation is in the sample. 


\section{Mass Spectrometry}

- Count atoms in a sample instead of radiation

- Good for materials with long half lives

-e.g., the average ${ }^{239} \mathrm{Pu}$ atom takes thousands of years before decaying, so you can have a lot of ${ }^{239} \mathrm{Pu}$ atoms without a lot of radiation. Better to count the atoms than the number of decays

- Each atom is ionized (given an electric charge) and accelerated towards a magnet - only ions with a specific mass will be turned by just the right amount so that they hit the detector

- By changing the magnetic field, you can 'select' different masses to be detected (e.g., 239)

- Great for ${ }^{239} \mathrm{Pu}$ because of low specific activity and no naturally occurring atoms with mass 239 . Less good for ${ }^{238}$ Pu because of higher specific activity and other atoms with mass 238 (e.g., ${ }^{238} \mathrm{U}$ )

- Sensitive enough to detect 'natural background' of ${ }^{239} \mathrm{Pu}$ in urine (about $1,000,000$ atoms per day) 


\section{Mass Spectroscopy}

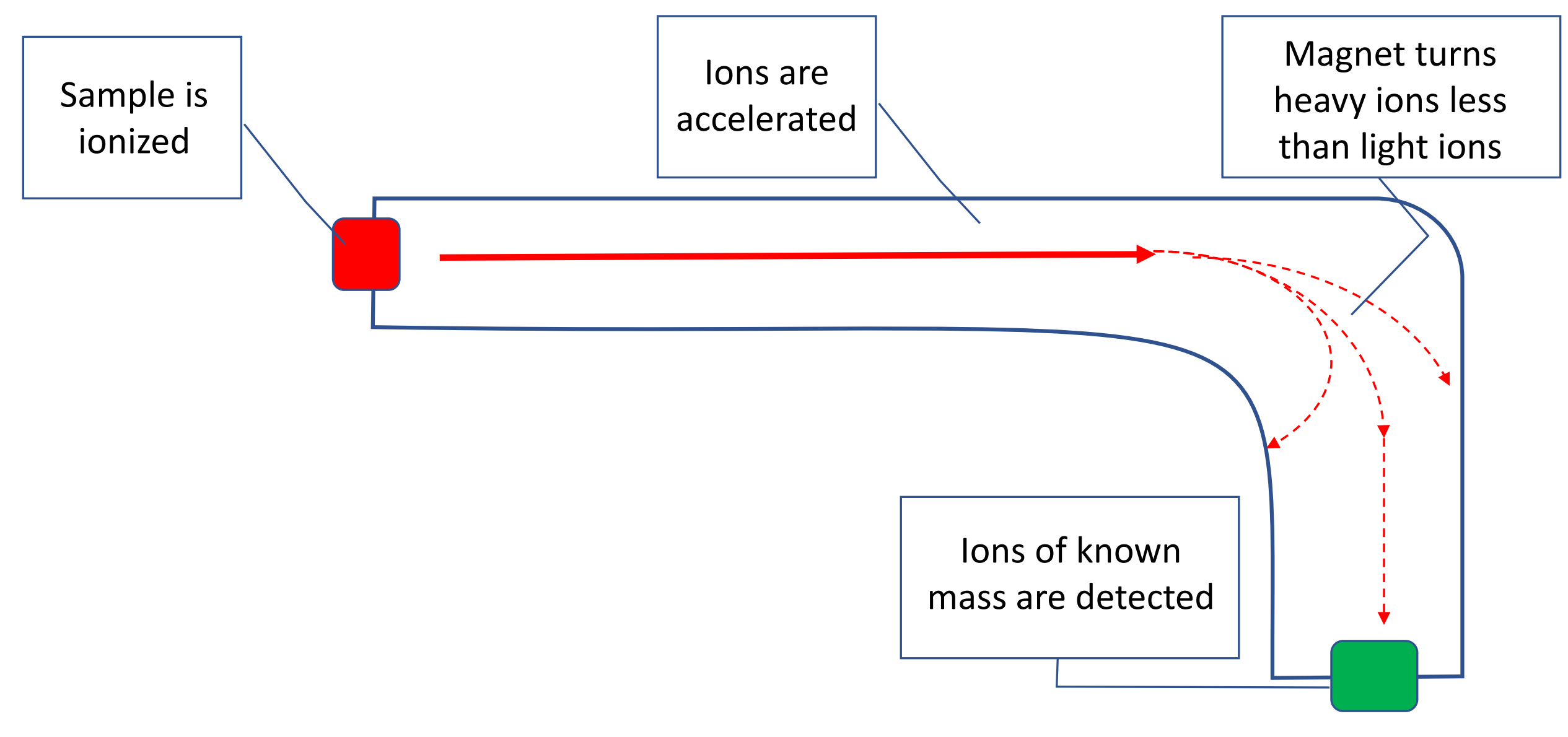




\section{Continuous Air Monitors (CAMs)}

- Continuously sample room air, alarm if dangerous radionuclides are detected

- An alpha detector (typically a scintillator) looks for sudden increases in activity

- Can be tricked by spikes in naturally occurring radon

- Because of radon, a relatively large amount of radionuclide in air required to set off alarm

- Alarm levels are usually conservative - i.e., lots of false alarms for each true detection 


\section{Fixed Air Samplers (FASs)}

- Pumps air through a filter, which is replaced weekly

- After waiting for radon to decay (half-life $\sim 30$ minutes), the filter is measured

- Much more sensitive than CAMs - can detect small leaks, potentially useful for retrospective dosimetry

- Cannot warn workers in advance - no alarm 


\section{Thermoluminescent Dosimeters and Film Badges}

- Used to detect neutrons

- Full of a low-density solid material - when neutrons pass through they create visible bubbles in the material

- The bubbles have to be counted to get a dose estimate. This used to be done manually, but now there are machines.

- You might encounter these at LANS (they look like a lemon cut in half) 


\section{Bubble Dosimeters}

- Used to detect neutrons

- Full of a low-density solid material - when neutrons pass through they create visible bubbles in the material

- The bubbles have to be counted to get a dose estimate. This used to be done manually, but now there are machines.

- You might encounter these at LANS (they look like a lemon cut in half) 


\section{Homework Questions}

- You dropped a tiny sample containing a photon emitter, and now you can't find it. What kind of detector would you use to look for it?

- You are cleaning a surface contaminated with alpha-emitting radionuclides. What kind of detector would you use to find contaminated areas?

- Why is mass spectrometry better for Pu-239 than it is for Pu-238?

- You are in a room in TA-55 when some material escapes a glovebox. Soon, an alarm goes off warning you of radionuclides in the air. What device is alarming?

- You are going to enter an area where you might be exposed to neutron and photon radiation. What kind of detectors should you have on you? 


\section{Plutonium}

Presented by John Klumpp, PhD, CHP 


\section{Intro to Plutonium}

- Man-made element - no longer exists naturally on Earth

- Two important isotopes $-{ }^{238} \mathrm{Pu}$ for heat sources and ${ }^{239} \mathrm{Pu}$ for weapons

- Both are primarily alpha emitters, and most dangerous if taken into the body

- Large quantities of either can produce lots of neutrons, so neutron dosimetry may be needed as well

- ${ }^{239} \mathrm{Pu}$ often contains ${ }^{241} \mathrm{Am}$, which emits relatively high-energy gammas in addition to alphas, making it more of an 'external' hazard 


\section{Intro to Plutonium}

- ${ }^{238} \mathrm{Pu}$ and ${ }^{239} \mathrm{Pu}$ are like liquor and beer, respectively - they emit the same types of radiation, but ${ }^{238} \mathrm{Pu}$ is much more concentrated

- This makes ${ }^{238} \mathrm{Pu}$ more hazardous for a couple of reasons:

-Smaller quantity of material needed to give a particular dose

-Small amounts of material get hot - can melt goves

-Neutrons can damage equipment and containment devices (e.g., glovebox gloves, washers in lines.

-Tiny particles can fly around under their own power, making them hard to contain 


\section{Plutonium as an Internal Hazard}

- The alphas emitted from Pu have similar energy to those from radon and radon daughters (naturally occurring radionuclides)

- These alphas are not energetic enough to penetrate your skin - you could hold a $15 \mathrm{lb}$ ball of ${ }^{239} \mathrm{Pu}$ in your hand without being harmed

- However, in the body small amounts of Pu can be toxic 


\section{What Pu Does in the Body}

- Starts off at the deposition site - e.g., the lungs or wound - and stays there until it dissolves into the blood

-Dissolution can happen very fast (seconds) or very slowly (years)

- Dissolved Pu stays in the blood for about a week. A tiny amount gets filtered out by kidneys and excreted in urine

- It then gets absorbed by the organs and tissues - almost all in the bones and liver.

- Stays in the body for decades, so we calculated the total dose the intake will cause over 50 years after it happens (CED(50))

- Radiation can't escape the body, but Pu can be detected in urine and feces 


\section{Intake vs. Dose}

- Intake is introduction of radionuclide into the body

- Dose is amount of radiation released by radionuclides in the body

- Doses from radionuclides in the body are called internal doses:

-external doses are caused by sources outside the body (e.g., an x-ray machine) 


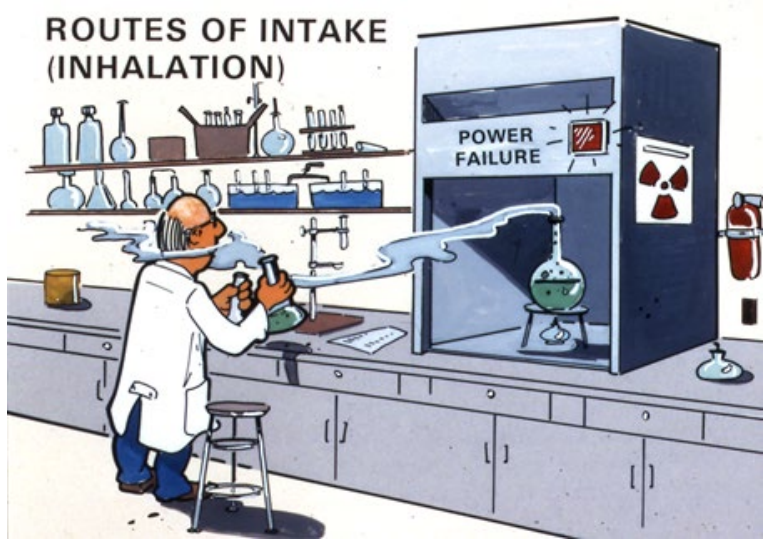

- Most common cause of intakes at the lab

- Most natural background comes from inhaling radon

- Detected by air monitors, nasal swabs

ROUTES OF INTAKE (WOUND)

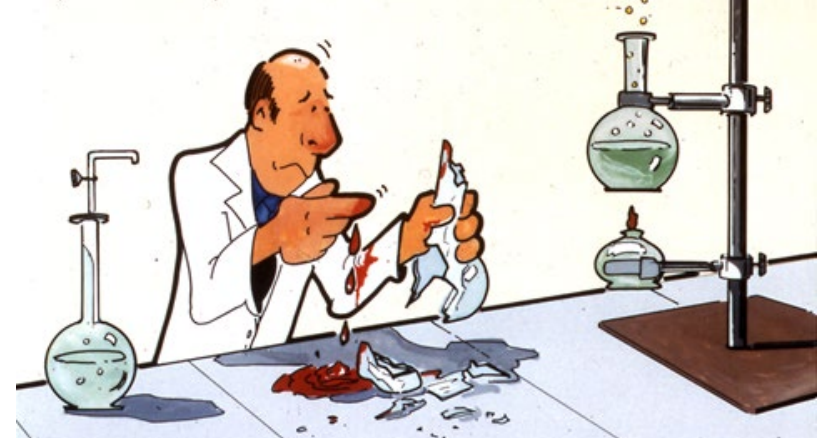

-e.g., injury by contaminated tool or chemical containing dissolved Pu

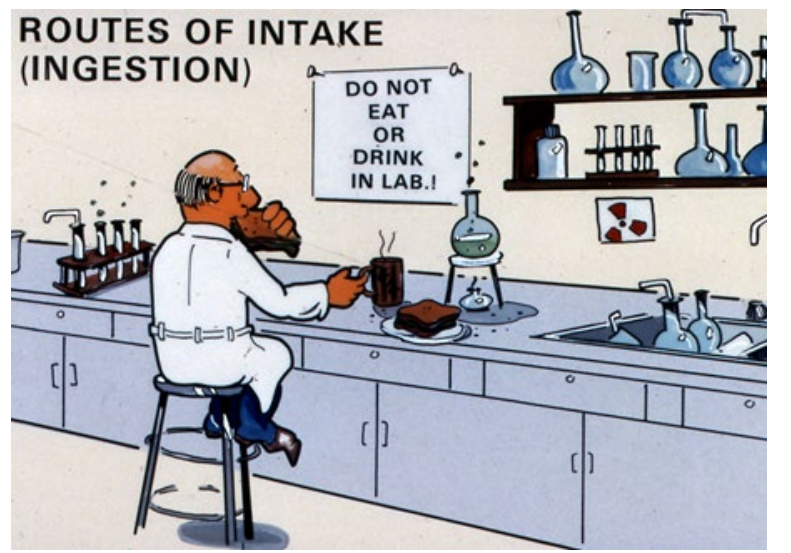

- Low absorption for Pu and Am, not usually a concern

- Next largest contribution to natural background comes from ingesting radionuclides in food (e.g., ${ }^{40} \mathrm{~K}$ in bananas) 


\section{Pu at the Deposition Site - Lungs}

- Inhaled Pu clears the lungs by two mechanisms which occur at the same time dissolution and mechanical transport

- Pu deposited in the upper respiratory tract (bronchi and above) is rapidly transported to the mouth and swallowed - this can be detected in fecal excretion

- Pu deposited in lower respiratory tract is more likely to be transported to the thoracic lymph nodes where it stays for a long time

- Low solubility inhaled Pu can give a significant dose to lungs 


\section{Pu at the Deposition Site - Wound}

- There is a tremendous variety of Pu wounds - cuts, scrapes, burns, punctures, etc.

-Some or all Pu can be dissolved/injected into the blood immediately -Some or all Pu can remain at the deposition site indefinitely

- Wound can be very serious (Pu not withstanding) and need immediate medical attention

- Some wounds can be so subtle they are not noticed at all

- If significant Pu remains in wound, a doctor may try to remove it before it can dissolve into the blood (procedure is similar to removing an unwanted mole) 


\section{Pu in the Blood}

- Pu dissolves into the blood as a Pu-citrate molecule - it's behavior no longer depends on the original chemical form

- Pu very quickly binds to transferrin, a molecule intended to transport iron into cells

- Transferrin isn't very good at bringing Pu into cells, so it keeps it circulating in blood much longer than other actinides like Am which don't bind to it

- A tiny amount of Pu in blood is filtered by kidneys and can be detected in urine

- After about a week, most of the Pu has left the blood and is bound to the liver and bones

- Some Pu gets metabolized by the liver which sends it to the GI tract and can be detected in feces 


\section{Pu in the Liver and Bones}

- Pu bound to the liver and bones stays for many decades

- A very small amount is 'recycled' back into the blood, so it can still sometimes be detected in urine

- The Pu decays very slowly, releasing alpha particles which are absorbed within 40 microns of the decayed atom - other organs and tissues are not affected

- Fraction which goes to liver vs. bone is almost random from person to person (can be 90-10 either way, or 50-50). Diseased livers absorb less Pu.

- Pu seems to migrate slowly from liver to bones over many decades 


\section{Detecting Pu Intakes}

- Alphas from Pu don't leave the body, so intakes have to be inferred or detected indirectly

- Intakes can be inferred from CAM alarms, Pu detected in FAS filters, facial or skin contamination, and positive nasal swabs.

- These are not good at predicting size of intakes

- Intakes usually only detected via bioassay (at LANL, urine measurements)

- Wound measurements can also detect intakes 


\section{Bioassay Thresholds at LANL}

\begin{tabular}{|c|c|c|}
\hline Indicator & Diagnostic Bioassay & Prompt Action Bioassay \\
\hline Air Concentration & $\begin{array}{l}40 \text { DAC-hr (or any CAM } \\
\text { alarm) }\end{array}$ & 200 DAC-hr \\
\hline $\begin{array}{l}\text { Nasal Swipe (sum of both } \\
\text { nostrils) }\end{array}$ & $75 \mathrm{dpm}$ & $400 \mathrm{dpm}$ \\
\hline Facial or skin contamination & $1000 \mathrm{dpm}$ & $5000 \mathrm{dpm}$ \\
\hline Wound contamination & Any positive result & $0.2 \mathrm{nCi}$ \\
\hline
\end{tabular}




\section{Nasal Swabs vs. Dose}

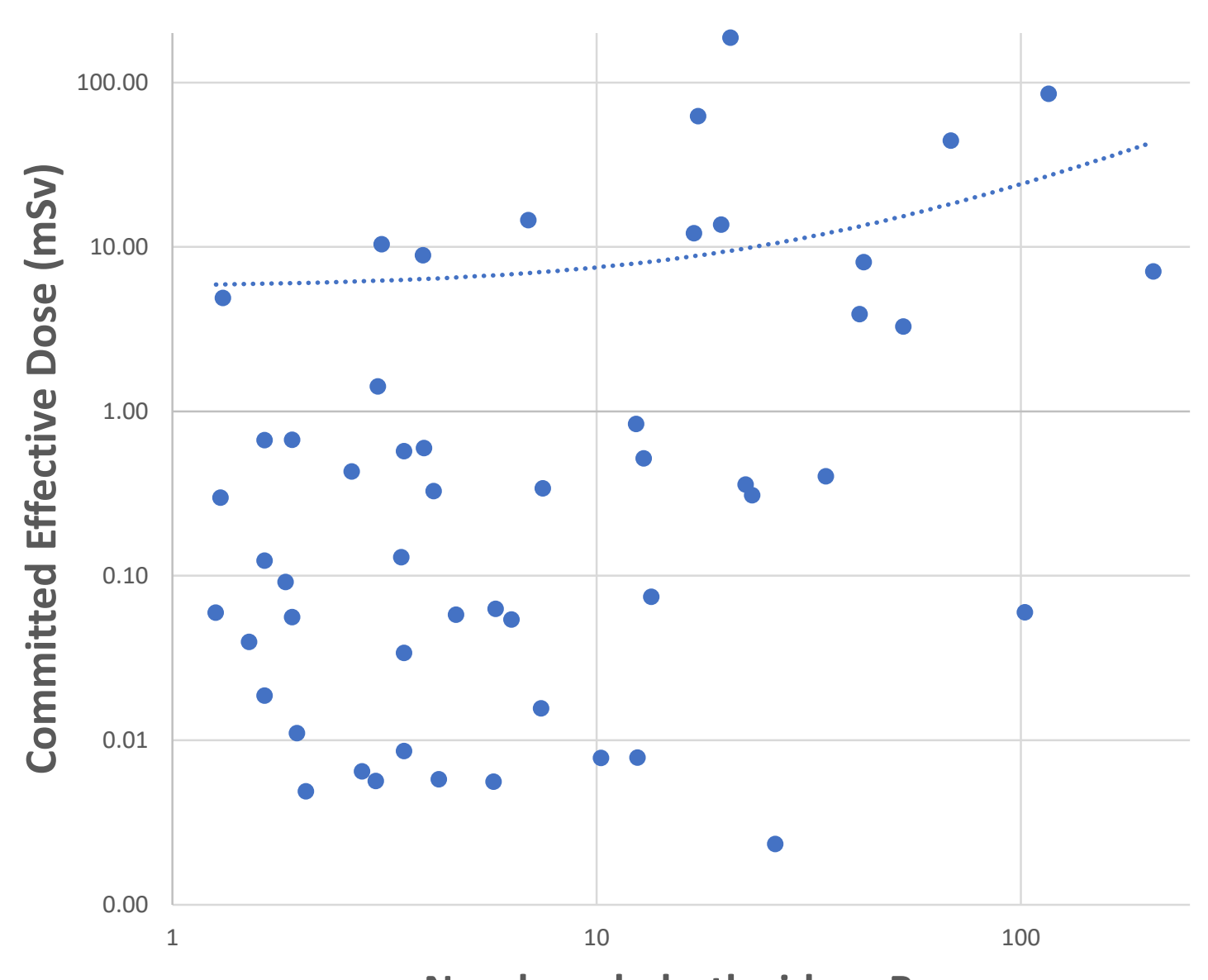

Nasal swab, both sides, Bq 


\section{Monitoring Workers with Bioassay}

- Bioassay of urine and feces is the most sensitive way to look for Pu in the body

- LANL Pu workers submit one or two urine bioassays each year for routine monitoring

- Bioassays are also collected after incidents (see thresholds table)

- Thresholds are intentionally very conservative - most bioassays confirm that no intake happened.

- Fecal measurements good for low solubility materials, but not fun

- Pu must be chemically separated from urine or feces before measurement. The process takes up to two weeks 


\section{Very Little Pu is Excreted in Urine}

- "High" sample is still tiny (e.g., $10^{-15}$ grams per day for ${ }^{238} \mathrm{Pu}$ ), or a few dozen decays in detected in a 24-hour measurement

- Samples sometimes get contaminated during analysis

- Up to 10 samples needed to confirm an intake

- Good new for people with big intakes - not enough is excreted to contaminate or endanger others

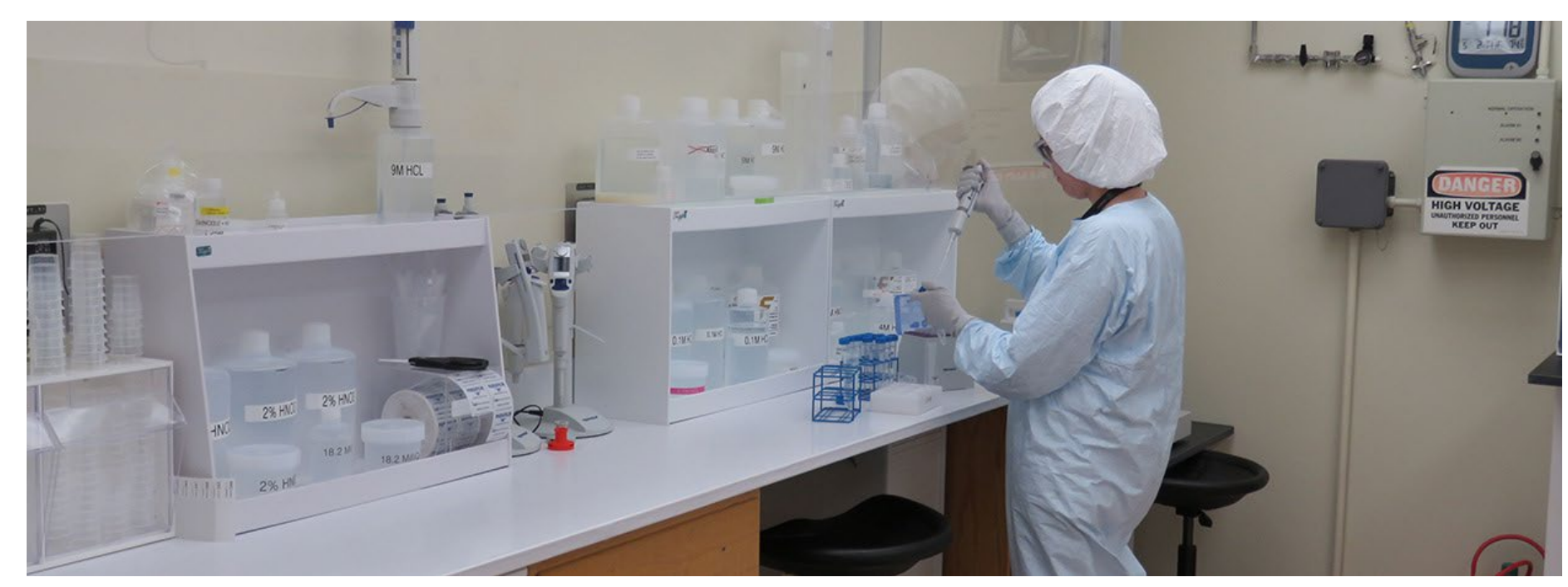




\section{An intake case}

Urinary Excretion Measurements of Pu-239

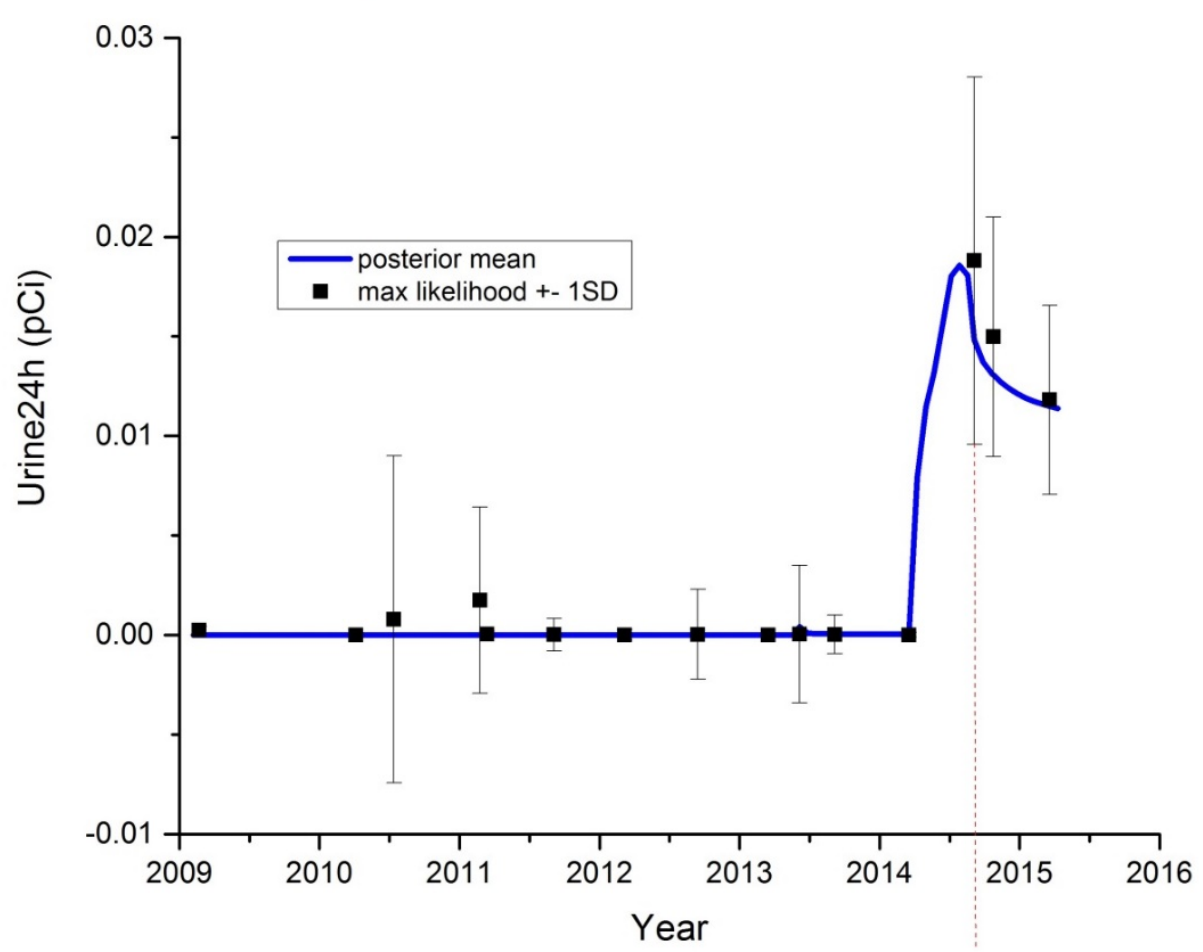

Urine bioassay data from a plutonium-239 intake. Each black square represents the result of a single urine sample. The thin vertical lines represent the statistical uncertainty on the measurement. The blue line represents the most probable value of the actual amount of radioactivity in urine being excreted over 24 hours. 


\section{An "no intake" case}

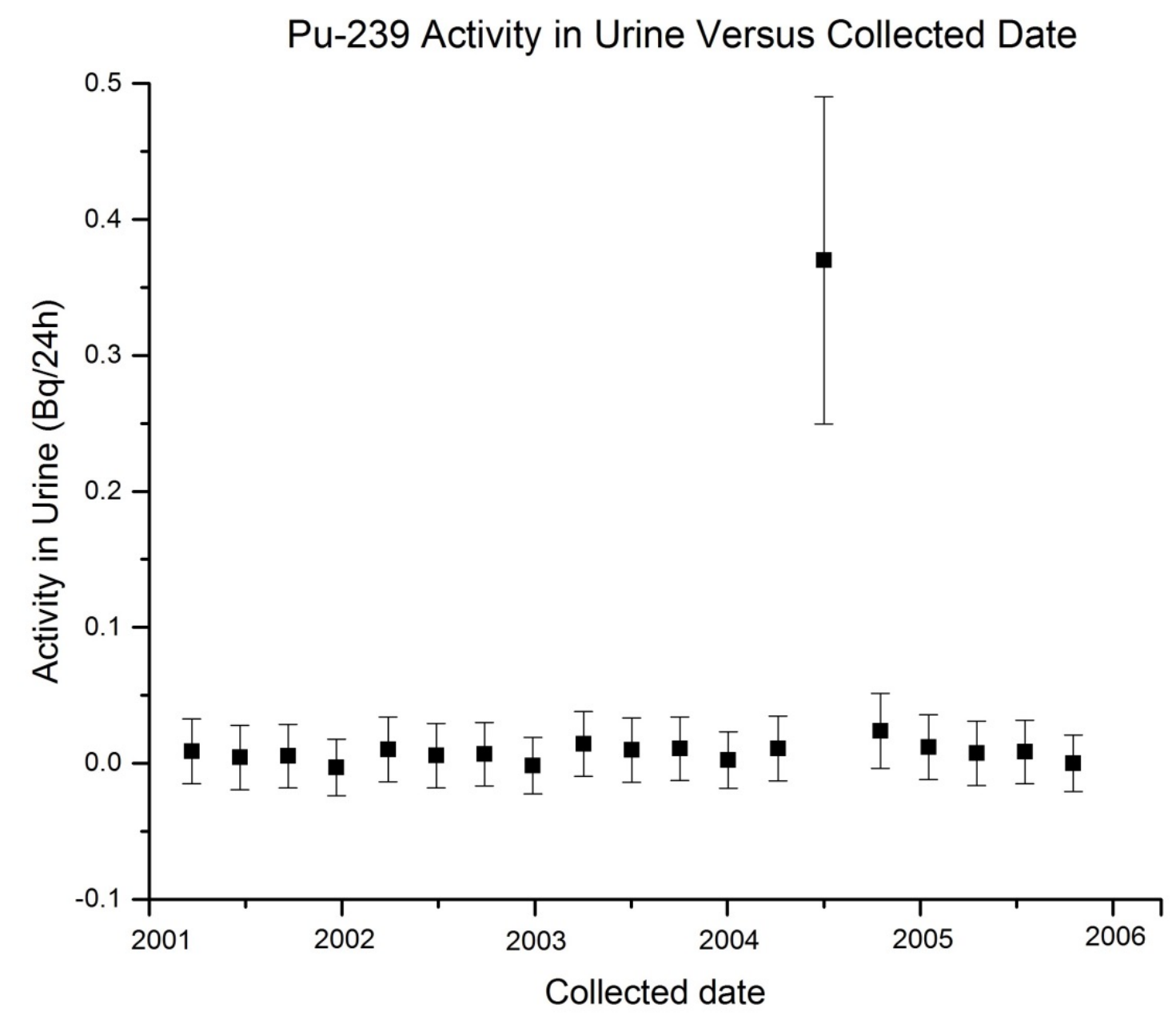




\section{Intake or No Intake?}

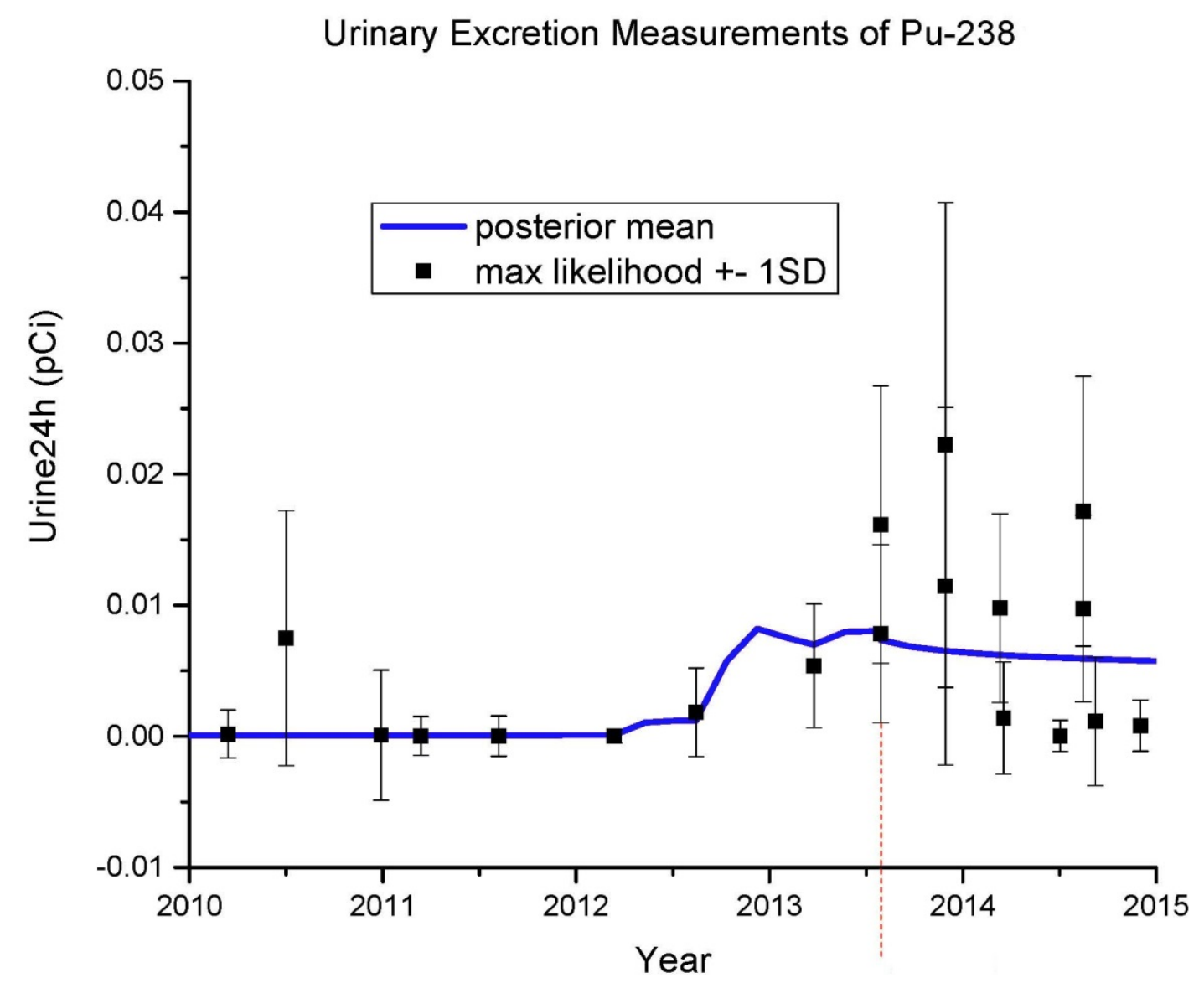




\section{Health Effects of Pu}

- Because of low dose rate, non-cancer effects have not been observed outside of the lungs. Analogy: a bottle of whiskey in one sitting vs. over 50 years.

- Radiation sickness impossible because Pu doesn't accumulate in bone marrow, intestines, or brain

- Cancer risk increases by $0.5 \%$ per 10,000 mrem (10 rem) (only for doses greater than 10,000 mrem)

- At LANL, 7 cases since 1985 larger than 10,000 mrem

- Largest internal dose ever at LANL: 312,000 mrem (312 rem) (1971)

- What is the risk from 1,000 mrem? 


\section{Psychological Effects}

- Months-long investigation and many bioassays -Anxiety has time to grow

-May imagine dose is larger with each sample request

- Results are difficult to understand, lots of uncertainty

- Actinides remain in body for life: worker takes the Pu home

- We make things worse 


\section{What happens if there is a big incident?}

- If there is a major incident - e.g., a contaminated wound or a large airborne release, worker goes to occupational medicine

- At occupational medicine, employee will be decontaminated if necessary, may receive a wound count

- If a wound, an attempt will be made to decontaminate. Doctor may recommend excision.

- Based on field indicators (CAM, nasals, wound counts, etc.), chelation may be offered

- Chelation delays dose estimate by several months 


\section{Chelation}

- Chelation removes plutonium and americium from the blood

- Materials only stay in blood for a few days before depositing in organs, so chelation usually only works shortly after intake

- Also removes important metals like, calcium, zinc, and manganese - you need to take a multi-vitamin with it

- Can cause side-effects like headaches, upset stomach, muscle cramps

- Not usually recommended for pregnant women (causes birth defects in dogs) 


\section{Chelation}

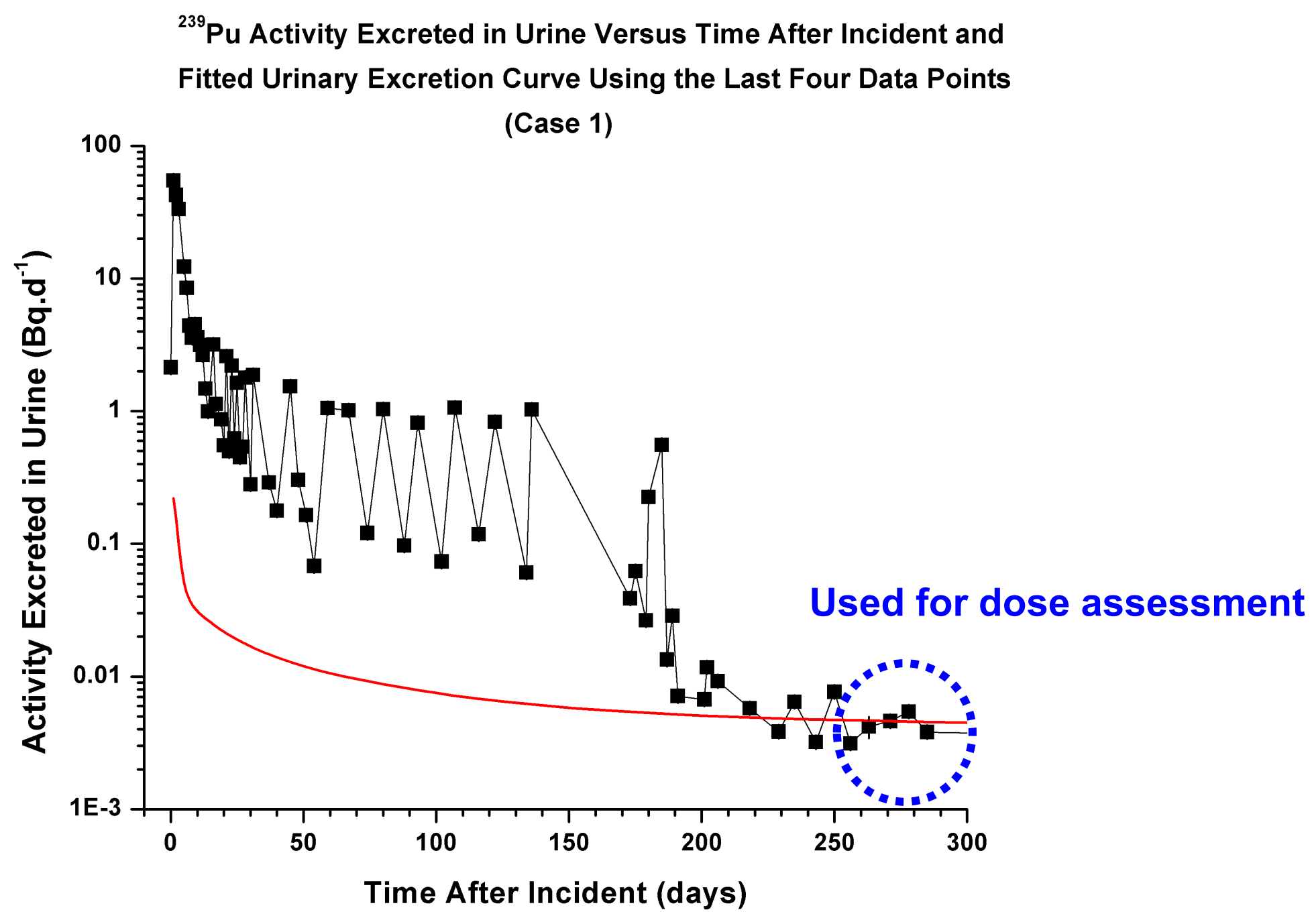




\section{Chelation}

${ }^{239} \mathrm{Pu}$ Activity Excreted in Urine Versus Time After Incident Showing the Chelation Treatment

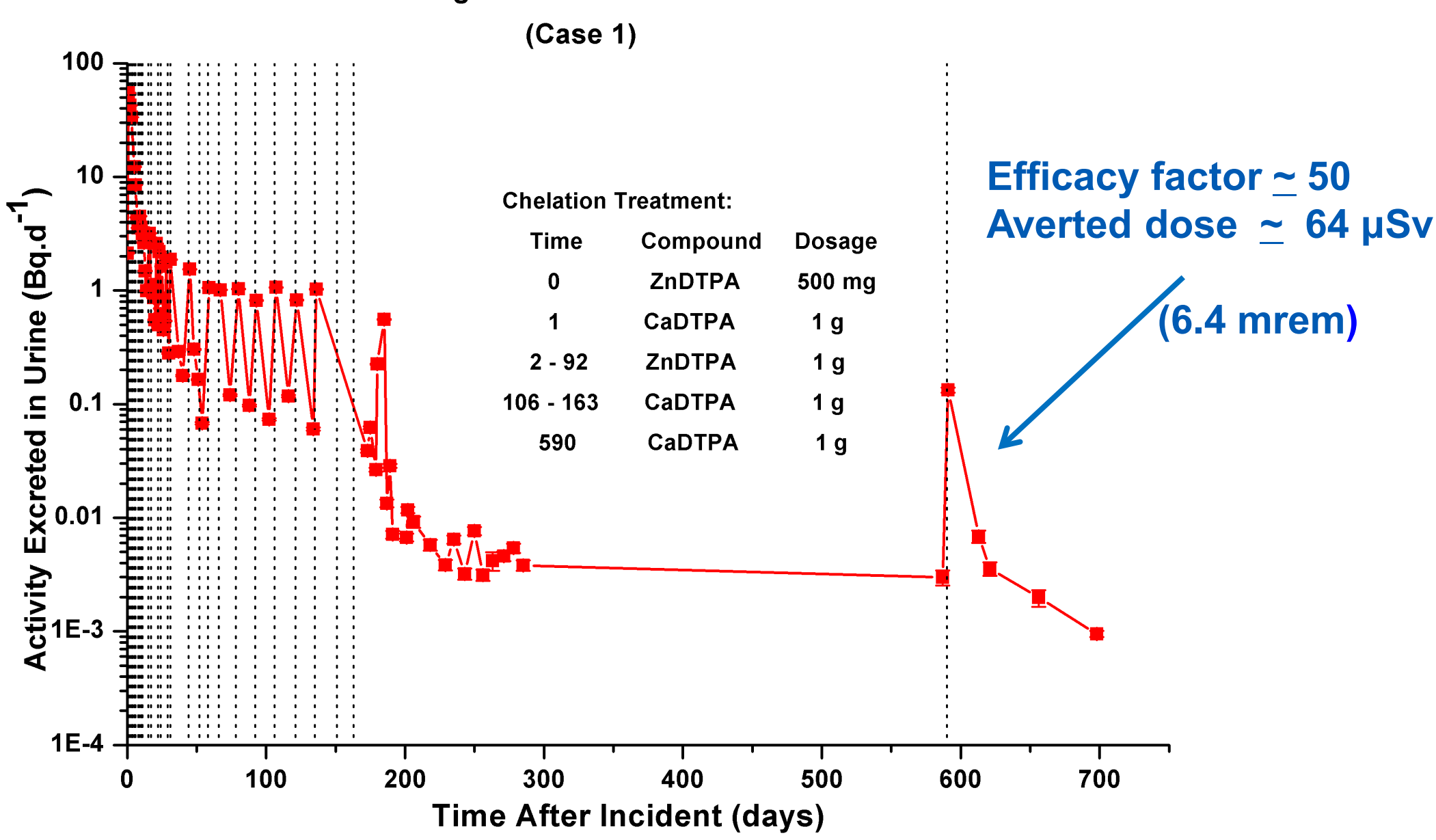




\section{Summary}

- Internal dosimetrists use urine bioassay to look for intakes

- It takes a lot of samples to confirm that an intake really happened

- Most intakes significantly smaller than natural background

- Most significant effect is usually psychological

- You can help protect your colleagues by providing support and understanding 


\section{Homework Questions}

- All other things being equal, which is more dangerous -1 gram of ${ }^{238} \mathrm{Pu}$ or 1 gram of ${ }^{239} \mathrm{Pu}$ ?

- All other things being equal, which is more dangerous, $1 \mathrm{Ci}$ of ${ }^{238} \mathrm{Pu}$ or $1 \mathrm{Ci}$ of ${ }^{239} \mathrm{Pu}$ ?

- You detect 7,000 dpm of alpha activity on your finger when exiting a plutonium lab. Your finger is easily decontaminated with gentle soap. Is bioassay recommended? What do you expect will happen to your finger?

- Your friend is diagnosed with thyroid cancer. She tells you that she has a large intake of plutonium 20 years ago. What would you tell her?

- You work in the plutonium facility for many years. During that time, you have to evacuate the laboratory multiple times because of a CAM alarm, and once were asked for bioassay samples after the alarm. However, your lifetime internal dose is listed as zero. How would you explain this?

- Explain the difference been an intake and a dose.

- You are placed on special bioassay following an 'elevated routine' bioassay samples. 6 months later, you are told that you had a plutonium intake sometime last year. Should you ask Occupational Medicine for chelation therapy to help remove the Pu from your body?

- You know that your friend has been placed on bioassay because of a possible plutonium intake. Lately, your friend has become less talkative and seems easily annoyed. What will you do? 
Other Radionuclides Monitored by Bioassay
and
Case Studies

Presented by John Klumpp, PhD, CHP 


\section{Other Radionuclides at LANL Require Urine Bioassay Monitoring}

- Employers must monitor employees for any radionuclides which are "likely" (i.e., possible) to cause doses of 500 mrem or more.

- We determine this based on the amount of material a worker handles at any given time

- Some of these radionuclides are best monitored by urine bioassay - At LANL, plutonium, americium, uranium, and tritium - because most of the radiation doesn't escape the body 


\section{Americium}

- A lot like plutonium in terms of hazard, but also emits gammas

- Gammas make it an external hazard, also sometimes allow it to be detected in vivo (i.e., in the lungs via whole body counter)

- When ${ }^{239} \mathrm{Pu}$ is chemically extracted from a reactor, some ${ }^{241} \mathrm{Pu}$ is removed with it

- ${ }^{241} \mathrm{Pu}$ is a beta emitter (not very dangerous), decays with $14-y e a r$ half-life to ${ }^{241} \mathrm{Am}$; WG Plutonium becomes more hazardous with time

- Sometimes chemically extracted from aged Pu. Used in smoke detectors. 


\section{Uranium}

- Used in weapons, reactors, and other applications. Naturally occurring, found everywhere on earth. We all ingest significant quantities with our food and water.

- Natural Uranium is a mixture of many isotopes: $99.3 \%{ }^{238} \mathrm{U}, 0.7 \%{ }^{235} \mathrm{U}$, and $0.005 \%$ $234 \mathrm{U}$

- Sometimes the ${ }^{238} \mathrm{U}$ is removed from natural $\mathrm{U}$ to produced 'enriched uranium' (extra ${ }^{235} \mathrm{U}$ ) and 'depleted uranium' (almost all ${ }^{238} \mathrm{U}$ )

- Natural Uranium is not very dangerous - about as toxic as copper - and the chemical toxicity is an issue before the radiotoxicity

- Enriched Uranium can be a significant radiation hazard (most of the radiation comes from ${ }^{234} \mathrm{U}$ ) 


\section{Uranium}

- Urinary excretion of natural uranium varies significantly from person to person and for a given person depending on diet

- Only very large spikes of natural uranium can be confirmed as occupational exposures

- Mass spec can be used to look for intakes of DU and EU by looking for changes in the ratio of ${ }^{235} \mathrm{U} /{ }^{238} \mathrm{U}$ - we can detect extremely small intakes/doses of these materials

- Urinary excretion can drop off very quickly, so monitored every two weeks 


\section{Tritium $\left({ }^{3} \mathrm{H}\right)$}

- Radioactive isotope of hydrogen used in weapons and special radiation detectors

- Often used as a gas, but rapidly enters water molecules as HTO in air

- Behaves exactly like water in the body - 'flushed out' in about 2 weeks

- Routine bioassays needed every other week because of short time in body

- Measured with LSC 


\section{Case Study: Tc-99 Incident}

- Tc-99 (HL: 211,000 years) accidentally released

- In spite of long half-life, Tc-99 is rapidly cleared by the body

- Ingestion of all of the released material: $<6$ rem

- Nonetheless, employee's car was confiscated and destroyed

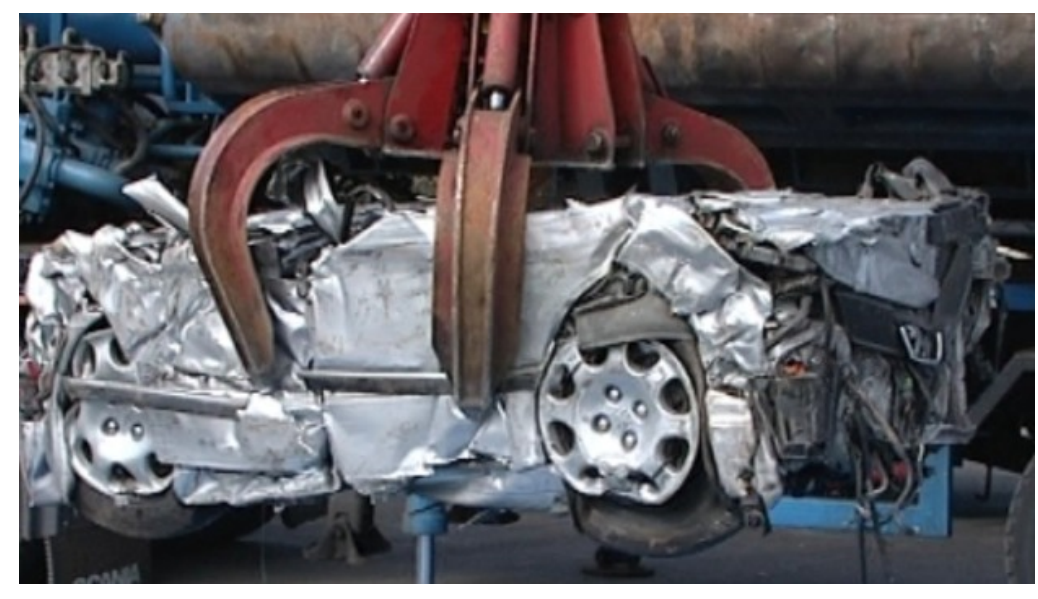




\section{Case Study: Hanford Tunnel Collapse}

- Spread of contamination at Plutonium Finishing Plant in June 2017.

- 31 workers tested positive for Pu-239, largest 50-year CED estimated 10 mrem.

- Excerpts from NBC King 5 local news coverage:

"...worker tested positive for inhalation of the potential lethal nuclear isotope of plutonium."

“'I'm pissed. I'm scared, like we all are, that sooner or later... I'm going to end up with cancer,' said the contaminated worker."”

"Thirty-one [workers] inhaled plutonium, which emits alpha radiation, the worst kind of radiation to get inside your body."

"I was angry. You carry that with you the rest of your life. It's a cancer causer."

"When they told me it was like an x-ray, I said, that's not true. An x-ray does not stay with me forever and this will. I know that alpha is a cancer causer. They know better and they are minimizing it." 


\section{Case Study: LANL Plutonium Worker}

- LANL glovebox worker cut his hand with a plutonium contaminated knife

- Initial dose estimate: $300-1000$ rem. 2 surgical excisions, 1 year of chelation therapy

- "I was starting to look like a junkie with so many holes in my arms. [The doctor] gave me his card and said to show it to the police ... if they ever stopped me.

- "Even my best friend wouldn't come close to me for a couple of months, wouldn't even shake my hand... he was frightened of what I had gotten in me... so he would talk to me at a distance."

- "For about a month, I went around wearing a surgeon's glove to try to sweat out the surface contamination."

- "I used to understand the numbers when they were expressed in terms of body burdens. Now that they've changed the system, I'm really confused, and it makes me wonder whether they are telling me everything." 


\section{Case Study: LANL Plutonium Worker}

- "The chelation did get to me. It made me kind of shaky and upset my stomach. I don't know if it was just the stress or the calcium in the chelation agent. Even to this day, if I take a multivitamin with calcium, I get a little shaky for an hour or so."

- "[When my wife got pregnant] I was concerned that the radiation might have affected the baby. That was a pretty stressful time. My son was born, and he was fine."

- "During the first year.. It felt like almost too much with all the wound counting and the urine analysis. But after that, I always wanted to hear more... I was hoping that if a similar thing did happen to someone else, they could use my experience to help that person."

- "If I catch a baseball it hurts near the area of the wound because they took out all the fatty tissue around it. But there's nothing really wrong with it." 
A Longer Case Study: A Contaminated Wound at LANL 


\section{The Incident}

- On Saturday (day 0), employee was replacing a glovebox cable

- Worker felt left glove breach and "a poke" on left ring finger

- $2 \mathrm{k} \mathrm{dpm}$ detected on ring finger, deconned to NDA* with tape press and gentle scrubbing

- Worker denied feeling anything touch skin

- Follow-up bioassay ordered Monday (day 2)

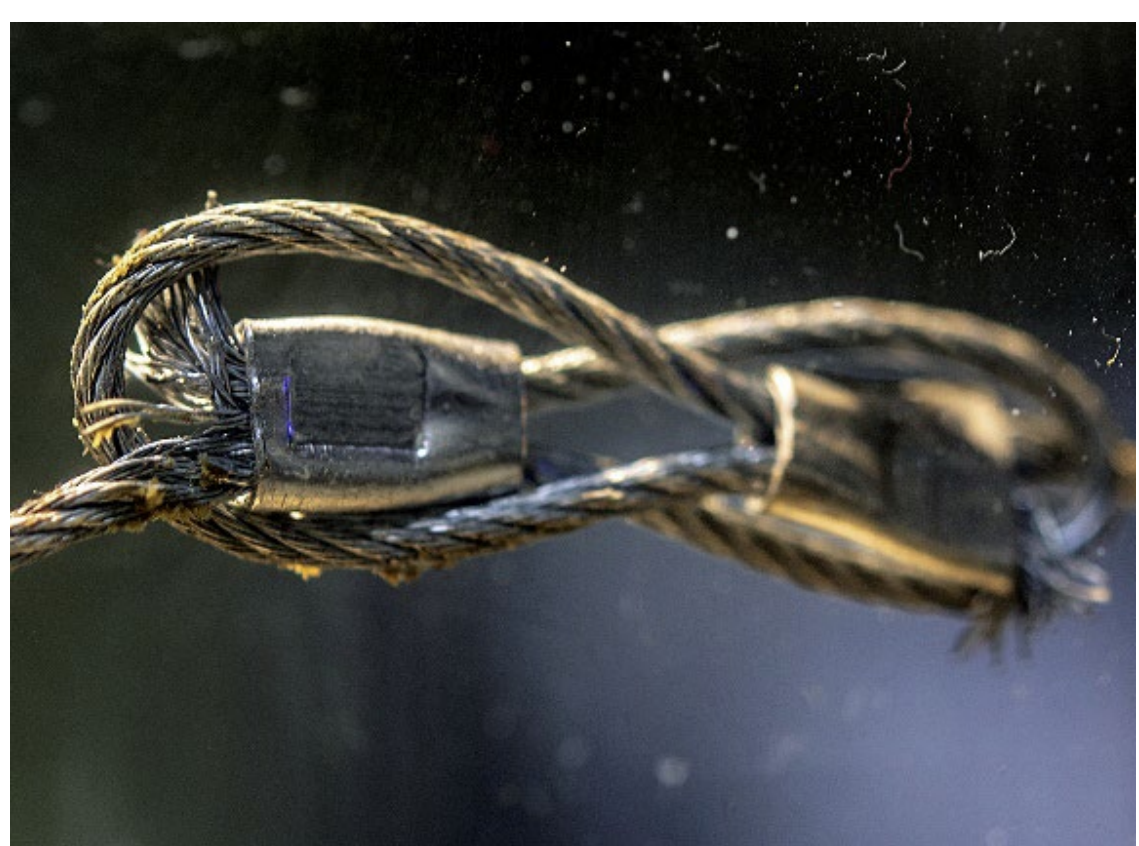




\section{Follow-up}

- Bioassay kit returned Thursday (day 5)

- Internal dosimetry received preliminary notification of elevated sample the following Thursday (day 12)

- Only known plausible $\mathrm{MOI}$ is inhalation - estimated dose as high as $100 \mathrm{rem}$

- ID suspects contaminated sample, but notifies RP personnel, orders additional bioassay samples

- An HP who is more familiar with circumstances requests a wound count 


\section{Follow-up (continued)}

- Wound count measures $\sim 150-244 \mathrm{~Bq}$ in the wound (depending on depth)

- Untreated dose estimate revised to $O(10 \mathrm{rem})$

- REAC/TS consulted, advised to begin chelation immediately with CaDTPA

- Hand surgeon contacted 


\section{Pre-Treatment Dose Estimate}

- Two measurements were collected before treatment - one urine bioassay and one wound count

\begin{tabular}{|c|c|c|}
\hline Model & Intake, Bq & $\chi^{2} / N$ \\
\hline WSW & 14300 & 165 \\
\hline WSM & 2800 & 87 \\
\hline WSS & 643 & 24 \\
\hline WSA & 392 & 0.61 \\
\hline WPA & 482 & 90 \\
\hline
\end{tabular}

- The pre-treatment measurements are consistent with NCRP "Soluble-Avid" (WSA) wound model

- Using this wound model, the intake is estimated to be $392 \mathrm{~Bq}$

- The effective (whole body) dose coefficient for this model is $4.18 \times 10^{-2} \mathrm{rem} / \mathrm{Bq}$

- The effective dose estimate (50 year CED) is 16.39 rem 


\section{Treatment Decisions}

- LANL policy (and standard practice) is for treatment decisions to be made by medical provider (MP) and employee

- Role of Internal Dosimetrist (ID) is to answer questions about rad risk

- Contaminated wounds are rare (luckily!), so MPs had no real-world experience with contaminated wounds or DTPA

-IDs were consulted heavily for treatment decisions

- Based on untreated dose estimate on the order of 10 rem, ID advised that rad risk was minimal, psychological concerns dominated risk 


\section{Treatment Guidelines for Pu}

\begin{tabular}{|c|c|c|}
\hline & Chelation & Recommendation \\
\hline NCRP 156 & 1 - 10 times annual limit averted & Unclear \\
\hline $\begin{array}{c}\text { CEC/DOE Guidebook } \\
\text { and DOE Good Practices for } \\
\text { OP in Pu Facilities }\end{array}$ & Offer if CED > 2 rem, Strongly Recommend if CED > 20 rem & Offer Treatment \\
\hline NCRP 161 (CDGs) & CED $>25$ rem & Do Not Offer Treatment \\
\hline $\begin{array}{l}\text { LANL Procedure } \\
\text { RP-SVS-ID-DP-06 }\end{array}$ & Consider if CED > 0.5 rem, Strongly recommend chelation if CED > 5 rem & Strongly Recommend Chelation \\
\hline
\end{tabular}

- Dose estimate from wound count and 1 bioassay: $C E D=10$ rem ( $2 x$ annual limit), CEqDBone Surface $=300$ rem (6x annual limit)

- LANL Procedure: "From the standpoint of radiation protection, therapy need not be considered unless prompt action bioassay has been assigned [indicated for potential doses $>0.5 \mathrm{rem}$ ]. If the potential dose exceeds $5 \mathrm{rem}$, chelation therapy is strongly recommended. Between these limits, treatment is regarded as optional. The affected person should always be made aware of the advantages and disadvantages of the treatment. 


\section{Risk/Benefit of Chelation Therapy?}

- Radiation risk is difficult to quantify

-Effective/equivalent doses control risk to population (prospectively)

-Regardless, rad risk was small to non-existent

-Virtually no risk of deterministic bone effects, despite exceeding bone dose limits

-Psychological harm likely to dominate adverse effects

- Effects of treatment even more difficult to predict:

-Side effects usually minor, anecdotal reports of serious side effects exist -Rad risk averted negligible

-Psychological effects will likely dominate, but will they be positive or negative? 


\section{Chelation Decision}

- Contacted REAC/TS, were advised to administer a course of chelation therapy Ca-DTPA for first treatment and Zn-DTPA subsequently

- ID and MP agreed to offer chelation but let employee decide because:

-Patient may feel empowered by being able to remove $\mathrm{Pu}$

-LANL seeks to minimize dose by any reasonable actions

- Advised employee that

-No harm from radiation likely if treatment declined

-DTPA would likely remove Pu

-Risks of side effects were small

- The employee requested chelation, so it was given 


\section{Wound Excision}

- Local rad effects possible - risk was physical as well as psychological

- Careful skin depth measurement would have helped estimate risk averted by excision, but also would have taken a long time

- Risk of serious side effects - infection, permanent pain or numbness, loss of function - essential to find a qualified surgeon

- MP advised excision, employee agreed

- Excision is a job involving Pu, blood, and a scalpel - very desirable to perform on site 


\section{Chelation in Retrospect}

- Relatively low efficacy (reduced CED by .008 Sv)

- Significant pain and bleeding from IV

- Cramps, nausea, headaches

- Significant, long-lasting drowsiness

- Employee appeared progressively more withdrawn and morose

- Months after treatment ended, employee repeatedly reported feeling "great since they stopped chelation" 


\section{Treatment Efficacy Estimate}

- The excised tissues were counted with a HPGe detector: $302.4 \mathrm{~Bq}$ removed

- Chelation removed an additional $20 \mathrm{~Bq}$ via urinary excretion

- Treatment removed a total of $322.4 \mathrm{~Bq}$, which corresponds to $13.46 \mathrm{rem}$ CED averted

- This led to an unofficial, preliminary post-treatment: $70 \mathrm{~Bq} / 2.93 \mathrm{rem}$ 


\section{Excision in Retrospect}

- Excision reduced systemic dose significantly

-Rad risk very low, but local radiation effects may have been prevented

- Excised tissue useful for determining averted dose

- Employee felt better knowing there was little Pu left in wound

- Finger was numb for months after excision, feeling slowly returned

- No infection, pain, or other complications 


\section{Post-Treatment Dose Estimate}

- The accepted method to estimate dose following chelation therapy is to analyze only the data considered unaffected by chelation (about 100 days after last treatment)

- At LANL, this is accomplished via a Bayesian analysis of the data using MCMC, and applying an informative prior on the intake

- The resulting dose estimate from this analysis was, $\operatorname{CED}(50)=3.60$ rem $(2.13,5.53)$ -This is remarkably similar to the dose estimate on previous slide (which was obtained using a different method and different measurements.

\begin{tabular}{l|c|c|}
\hline Organ & CEqD (rem) & Credible Limits (rem) \\
\hline Bone Surfaces & 118.5 & $(70.7,182.3)$ \\
\hline Liver & 25.1 & $(14.9,38.6)$ \\
\hline Red Marrow & 5.9 & $(3.5,9.1)$ \\
\hline Gonads & 1.6 & $(0.9,2.4)$ \\
\hline
\end{tabular}




\section{Dose Estimate Conclusions}

- Without treatment, the whole body effective dose estimate is 16.39 rem (50 year CED)

- After treatment (chelation and surgical excision) the dose estimate is 3.6 rem (50 year CED). The dose estimate to bone surfaces is 118.5 rem.

- Treatment reduced dose by approximately $80 \%$. Excision removed 302.4 $\mathrm{Bq}$, chelation removed approximately $20 \mathrm{~Bq}$.

- Because of the low dose rate, no deterministic effects are expected as a result of the bone dose. 


\section{Urine Bioassay Data}

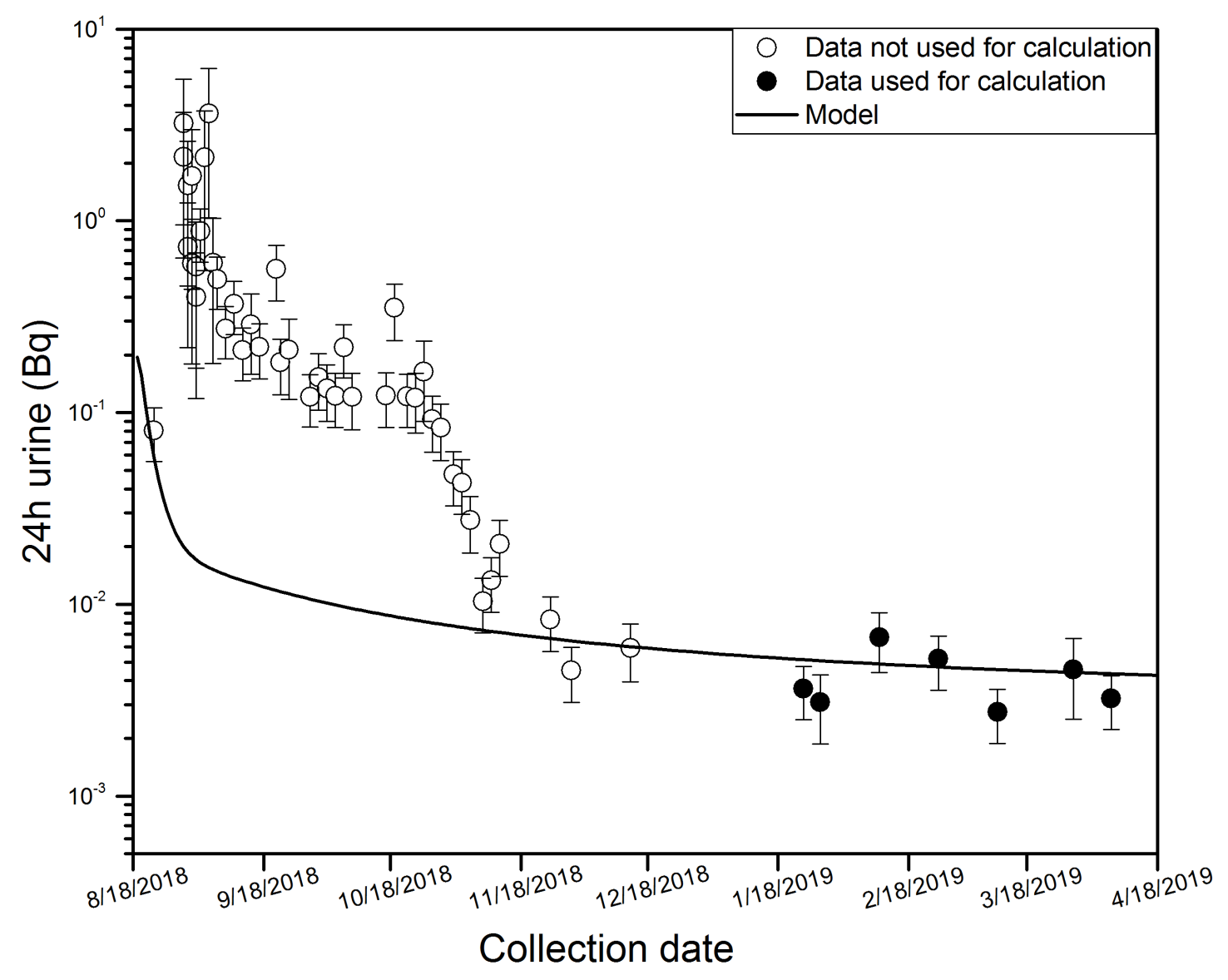




\section{Counting Statistics}

John Klumpp, PhD, CHP 


\section{Introduction}

\section{Counting Measurements}

- Count the number of events in a given time or space

- Examples:

-Radioactive decays in a time interval

- Stars in a region of space

-Animals in a geographic territory

-Mutations on a genome
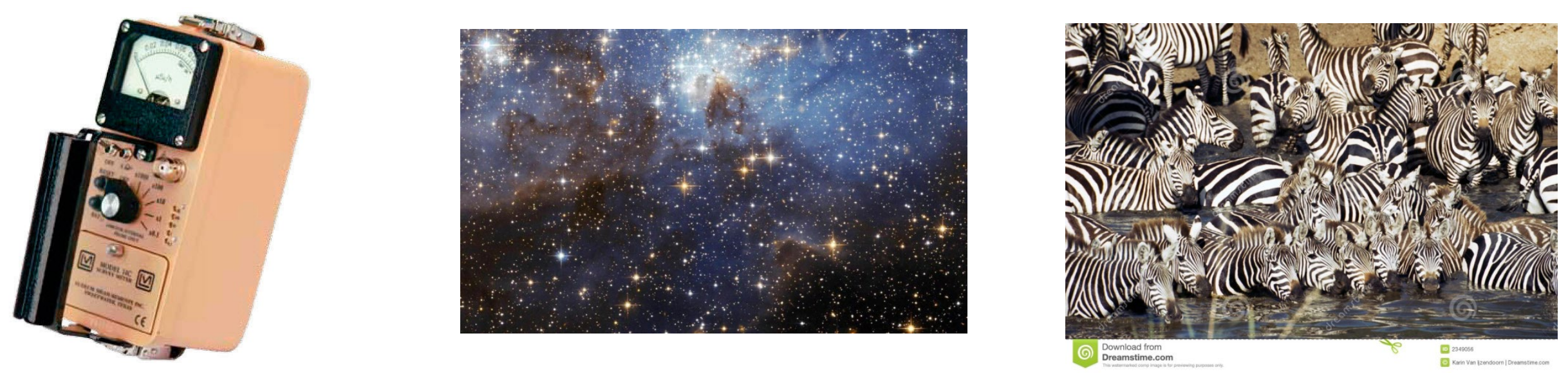


\section{Introduction}

\section{Common Questions}

- How many events are likely to occur in the next interval?

- Has the average number of events changed?

- How will I know if something I do changes the average number of events?

- Anything that answers those questions we call a detection system

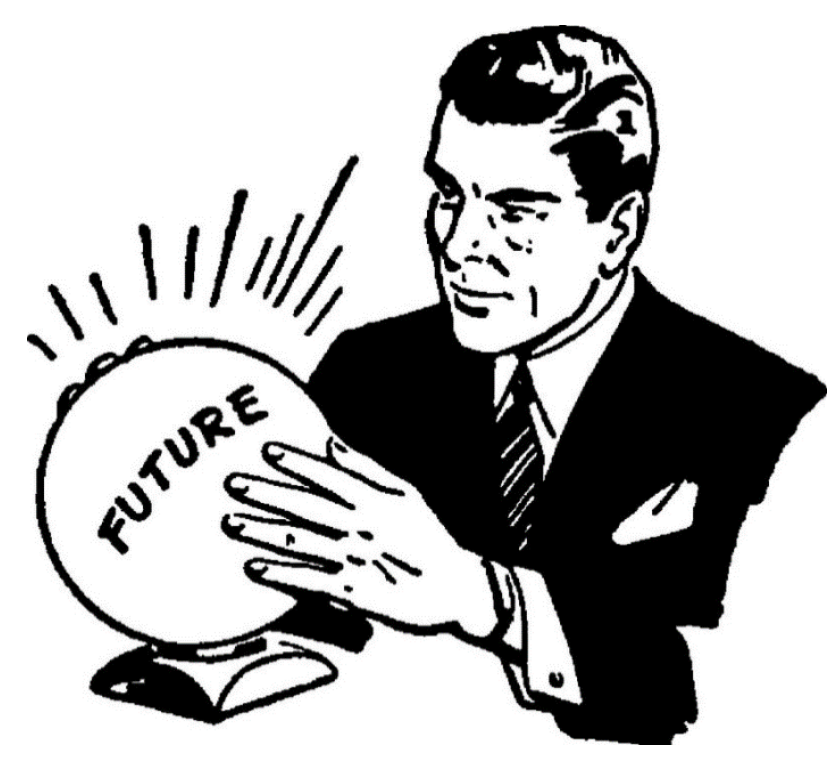




\section{Introduction}

Radiation Counting Measurements

- Counts come from a Poisson distribution, with mean count rate $\lambda$

- Detecting a radioactive source (e.g., a weapon, a calibration source, a gamma ray burst)

- Estimating source strength (e.g., medical imaging, dosimetry, decontamination)
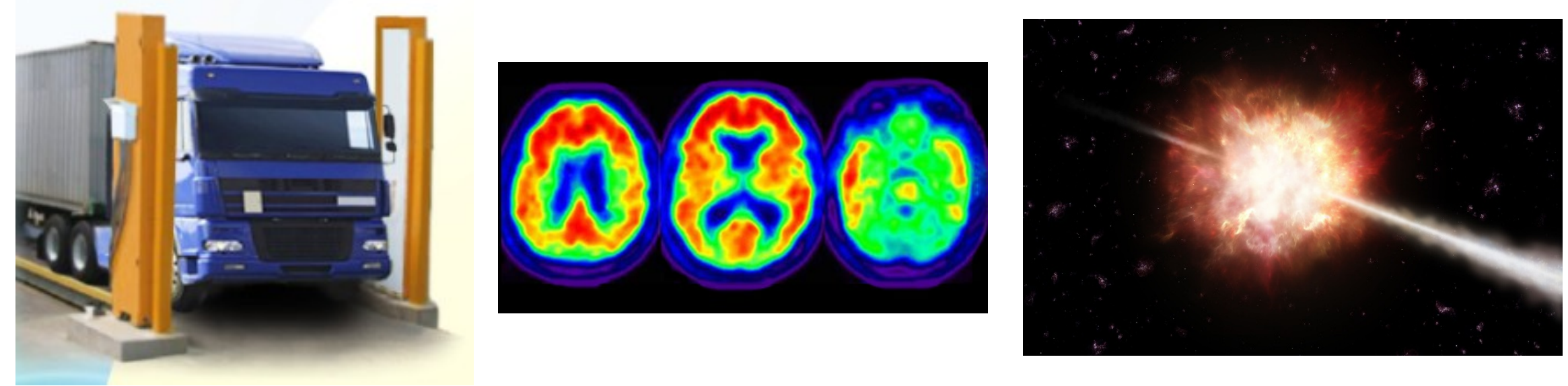


\section{Atoms Decay Randomly}

- An unstable atom has a fixed probability of decay at any instant. This is called the 'decay rate,' usually written as the Greek letter lambda $(\lambda)$

- If you have $N$ atoms, the decay rate is $N \lambda$ (e.g., 30 decays per second, $30 \mathrm{~Bq}$ )

- As atoms decay, $\mathrm{N}$ changes, so the count rate goes down. At a given time the number of atoms left is $\mathrm{N}=N_{0} e^{-\lambda t}$ and the decay rate is $\lambda \mathrm{N}=\lambda N_{0} e^{-\lambda t}$

- The average time a given atom takes to decay is $1 / \lambda$. An atom has a $50 \%$ chance of decaying in $\ln (2) / \lambda=0.693 / \lambda$. This is called the half-life, written $t_{1 / 2}$

- The half-life tells you how long it takes for half of a large collection of atoms to decay. After two half-lives, a quarter of the atoms are left 


\section{Let's talk about probability...}

- An approach to the unknown and making predictions

- How likely is it that something will happen? How likely is it that something is true?

-E.g., How many radioactive decays will we detect vs. how likely is it that this sample is radioactive

-We treat these different kinds of unknowns the same way

- Example: What are the chances that a coin is fair (i.e. equal chance of heads and tails)? What are the chances that when we flip the coin it will come up heads? 

we thing might happen/be true

- E.g., very simply probability distribution would be that there is a $50 \%$ chance that the coin will turn up heads and a $50 \%$ chance it will turn up tails

Simple Probability Distribution

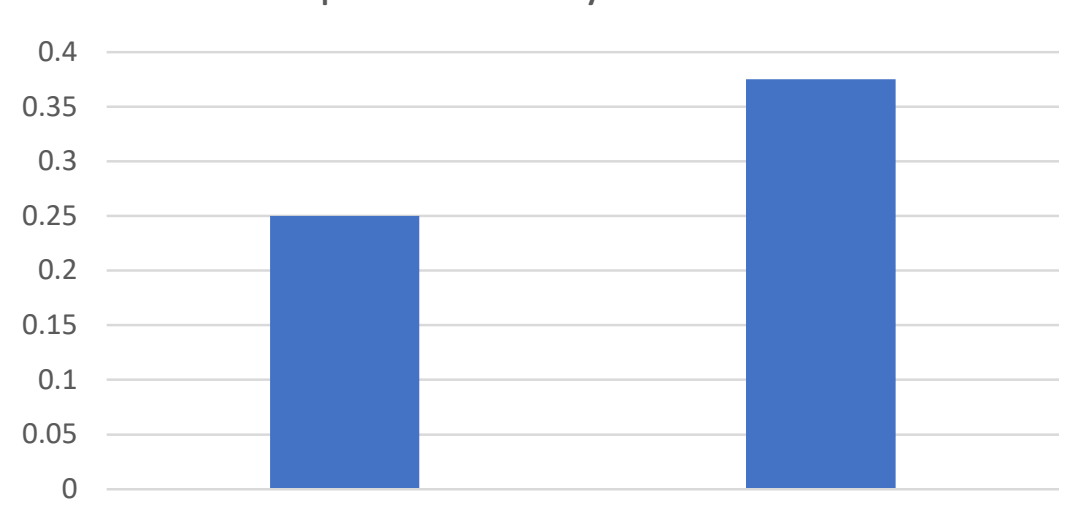

- Another example would be that there is a $20 \%$ chance of detecting 0 counts, $30 \%$ chance of detecting 1 count, $20 \%$ chance of detecting 2 counts $10 \%$ chance of detecting 3 counts, $10 \%$ chance of detecting more than three counts

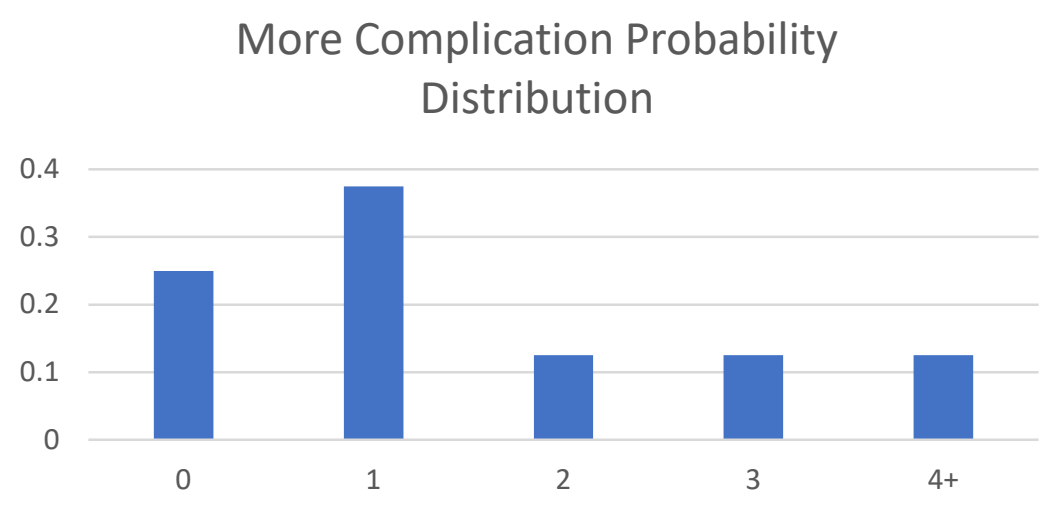




\section{Probability distributions}

- We could put a continuous probability distribution on the probability distribution of the count rate, or of the probability of a coin turning up heads on a given flip (the 'area' under part of a probability distribution is the probability of selecting a value in that range)

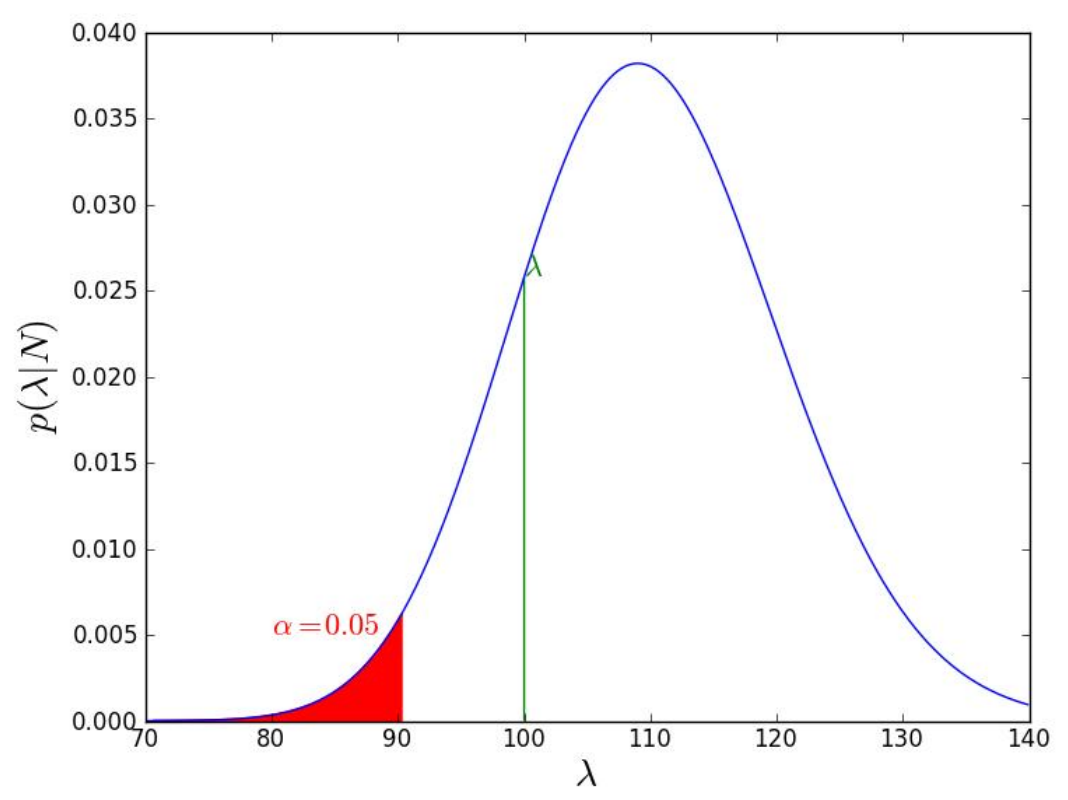

- The number of counts detected, and the number of heads, are random numbers which are 'sampled' from a probability distribution.

- We can treat the unknown probability of heads, and the count rate, as random numbers sampled from a probability distribution as well, even though they have fixed, true values. 


\section{Atoms Decay Randomly}

- An unstable atom has a fixed probability of decay at any instant. This is called the 'decay rate,' usually written as the Greek letter lambda $(\lambda)$

- The probability of the atom decaying in time $\mathrm{t}$ is $\mathrm{p}=1-e^{-\lambda t}$ (e.g., an atom might have a $50 \%$ chance of decaying in any given minute)

- Suppose you have lots of atoms. The most likely number of decays is $\mu=N p$ (the number of atoms times the probability of any one atom decaying during that time interval)

- Suppose you have two atoms (Atom A and Atom B) with $50 \%$ chance of decay in a minute. What are the odds of various outcomes?

$-25 \%$ chance they both decay

$-25 \%$ chance $A$ decays but not $B, 25 \%$ chance $B$ decays but not $A \rightarrow 50 \%$ chance one decays $-25 \%$ chance neither decays

- What if you have more atoms? For an arbitrary number of atoms, $\mathrm{n}$ :

Probability of $x$ decays $=\frac{n !}{x !(n-x) !} p^{x}(1-p)^{n-x}$ (Binomial Distribution) 


\section{What if the number of atoms is really big?}

- Atoms are small. Really small. So even a small amount of material has an enormous number of atoms (e.g., about $6 \times 10^{23}$ atoms in 1 gram of carbon)

- Luckily, the probability of any one atom decaying in, say, 1 second, is very small (if they all decayed at once you would have a nuclear explosion)

- The binomial distribution simplifies to the Poisson distribution:

Probability of $\mathrm{x}$ decays $=\frac{\mu^{x} e^{-\mu}}{x !}$, Standard Deviation $=\sqrt{\mu}$ 
The number of decays you detect is random
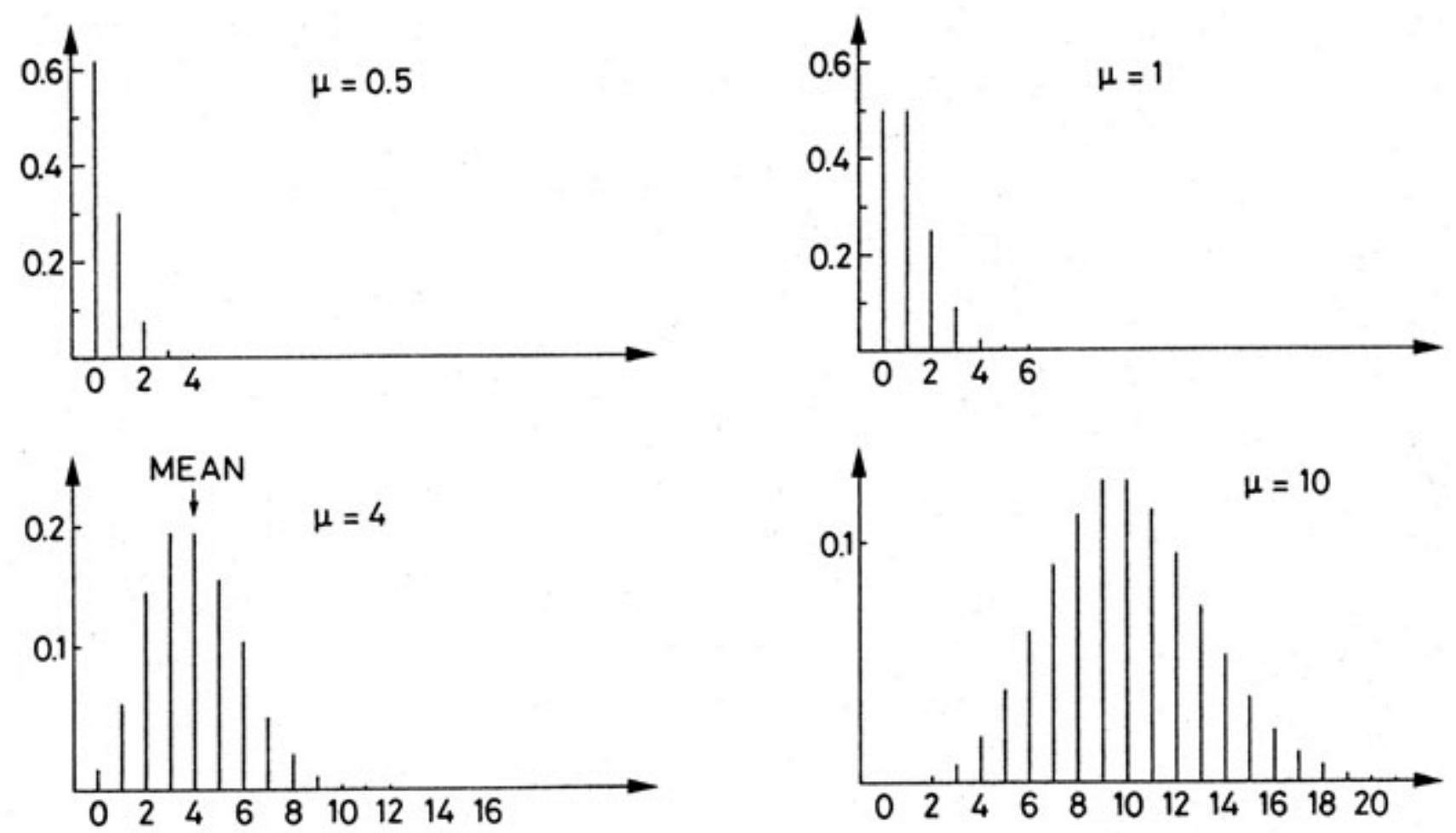
What if you don't know the count rate, or if it is changing randomly?

What we expect

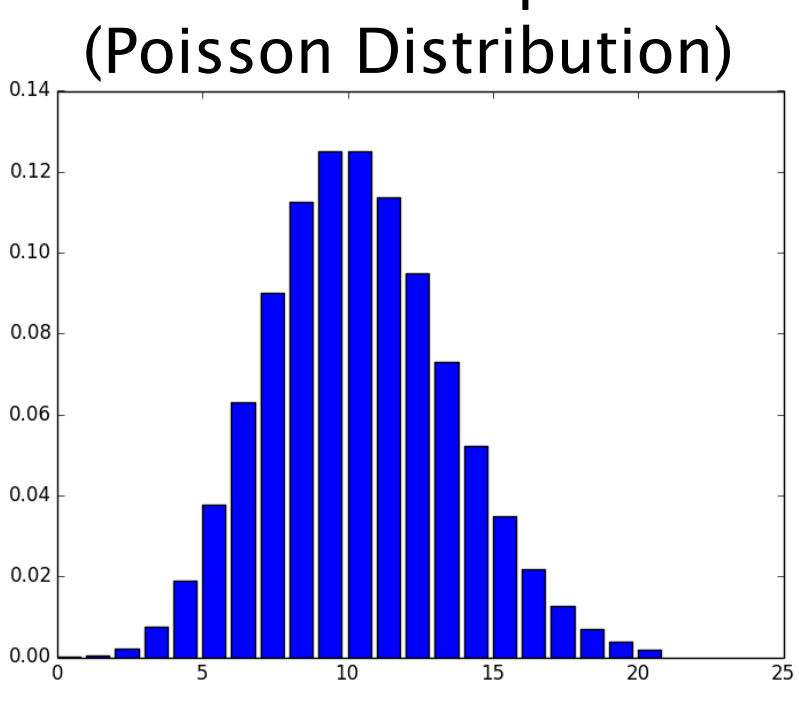

What we get

(Negative Binomial Distribution)

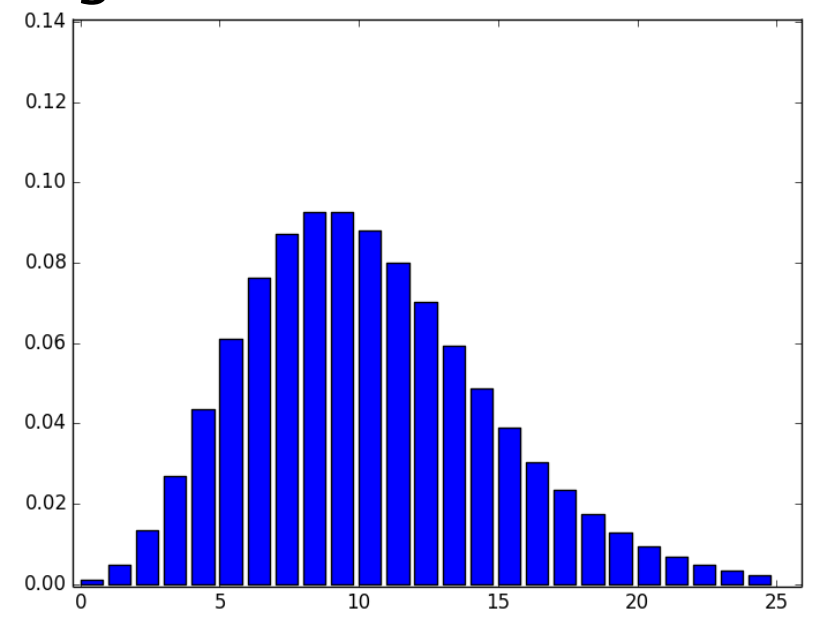


If the number of decays you detect is random, how do you figure out the 'true' mean decay rate?

- Short answer: measure longer

- Suppose the 'true' mean decay rate is 5 decays per second. The standard deviation is $\sqrt{\mu}=\sqrt{5}=2.2$. So you have a good chance of detecting anywhere between about 1 and 8 decays - in other words, your measurement is likely to very different from the average count rate.

- Suppose you do the measurement for 100 seconds. The most likely number of decays is 500 . The standard deviation is $\sqrt{500}=22$. So you are likely to measure between about 478 and 522 decays.

- Your estimated decay rate is likely to be between 4.78 and 5.22 decays per second 


\section{Detecting sources above background}

- Requires separate background and sample measurements

- But counts from background follow a Poisson distribution

- Typical approach: Step 1 is to guess what the background count rate/distribution is based on the background measurement. Step 2 is to calculate if the number of counts in the sample measurement is likely given the background distribution.

- There is always some probability that you are wrong (false positives and failed detections) 
The rate of false positives and failed detections depends on how many samples are 'positive'

1. Suppose we test 4000 people. Of those, 20 have had intakes.

2. Suppose test is right $95 \%$ of the time.

3. 218 people get positive results. Of those, 199 had no intakes.
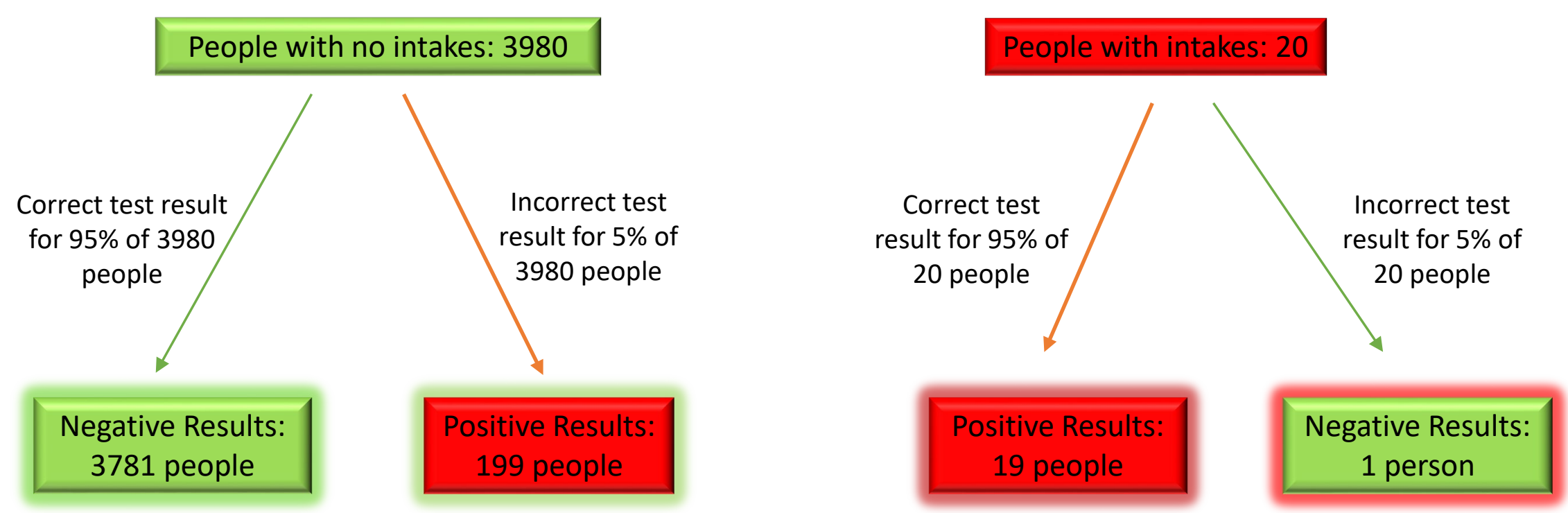

Even very accurate tests can lead to false alarms. In this case, even though the test was right $95 \%$ of the time, only about $10 \%$ of people who got positive test results actually had an intake! 
- Consider the following data (from Little 1982): $N_{B}=84$, $t_{B}=18.56 \mathrm{~s}$, $N_{G}=16$, $t_{G}=4.64 \mathrm{~s}$.

- Observed gross count rate: $3.5 \mathrm{cps}$

- Observe background count rate: 4.5 cps

- How to estimate probability there is a source? 
- Define a probability distribution for whether or not sample contains a source:

$$
p_{s}=0.5
$$

- Then a probability distribution for how strong source would be if it exists:

$$
\widehat{\lambda_{s}} \sim \operatorname{Uniform}(0,5)
$$

- Analyze with MCMC 
Example 1: Negative Observed Source

Count Rate

$\overline{\lambda_{S}}=0.083$,

$95 \%$ HDI $= \begin{cases}{[0]} & 87 \% \\ (0,0.68) & 13 \%\end{cases}$

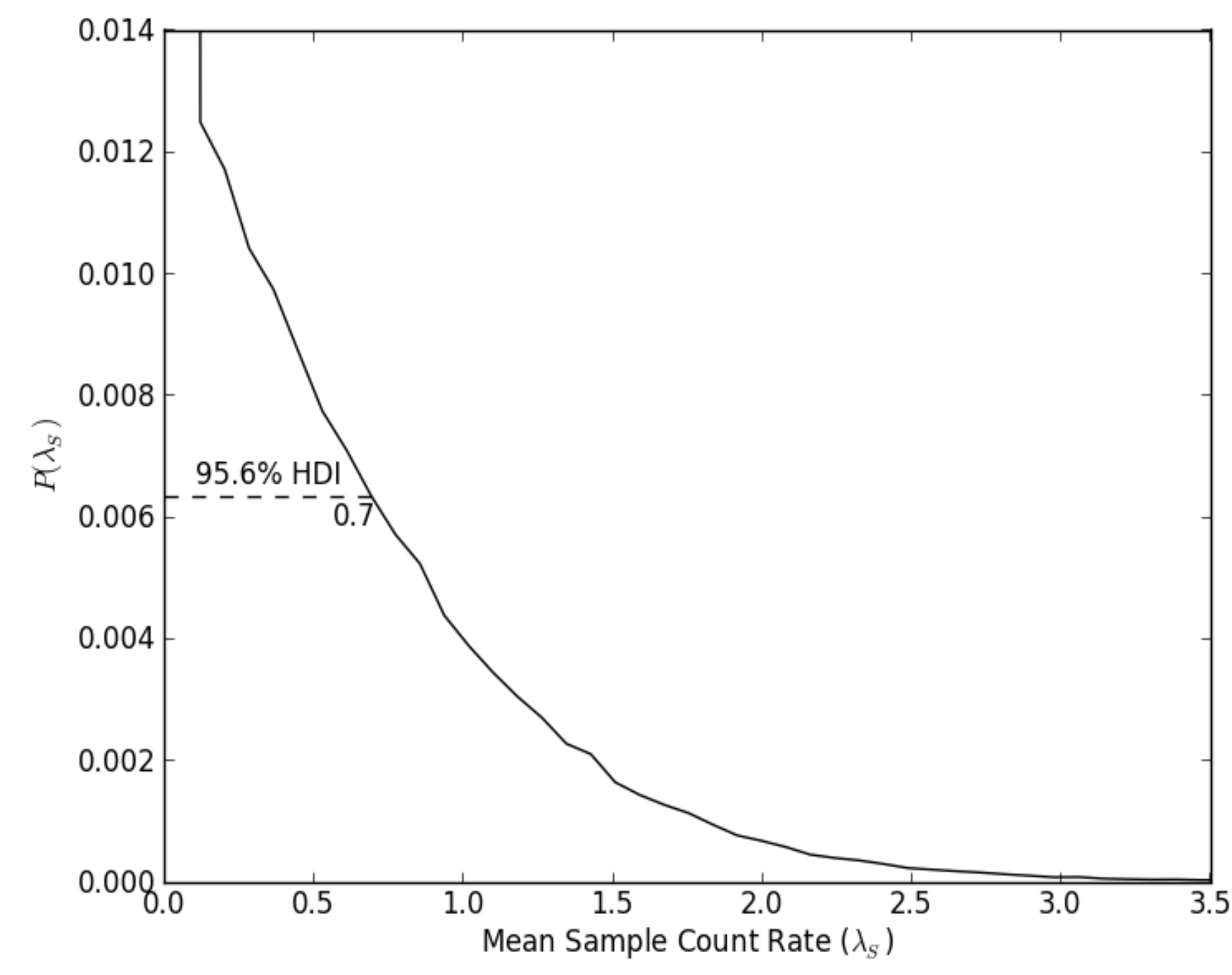




\section{Example 2: Strong Signal, Rare Source}

- Consider the following data:

$N_{B}=100$,

$t_{B}=25 \mathrm{~s}$,

$N_{G}=90$,

$t_{G}=15 \mathrm{~s}$.

- Observed gross count rate: 6 cps

- Observe background count rate: 4 cps

- How to estimate probability there is a source? 


\section{Example 2: Strong Signal, Rare Source}

- Suppose we know 1 in 20 samples contain a source: $p_{s}=0.05$

- Suppose we know sources are about $2.5 \mathrm{cps}: \widehat{\lambda_{S}} \sim \operatorname{gamma}(2.5,1)$

- Analyze with MCMC 


\section{Example 2: Strong Signal, Rare Source}

\section{Example 2 Results:}

$$
\overline{\lambda_{S}}=1.06 \text {, }
$$

$$
95 \% \mathrm{HDI}= \begin{cases}{[0]} & 46 \% \\ (0.9,3.1) & 54 \%\end{cases}
$$

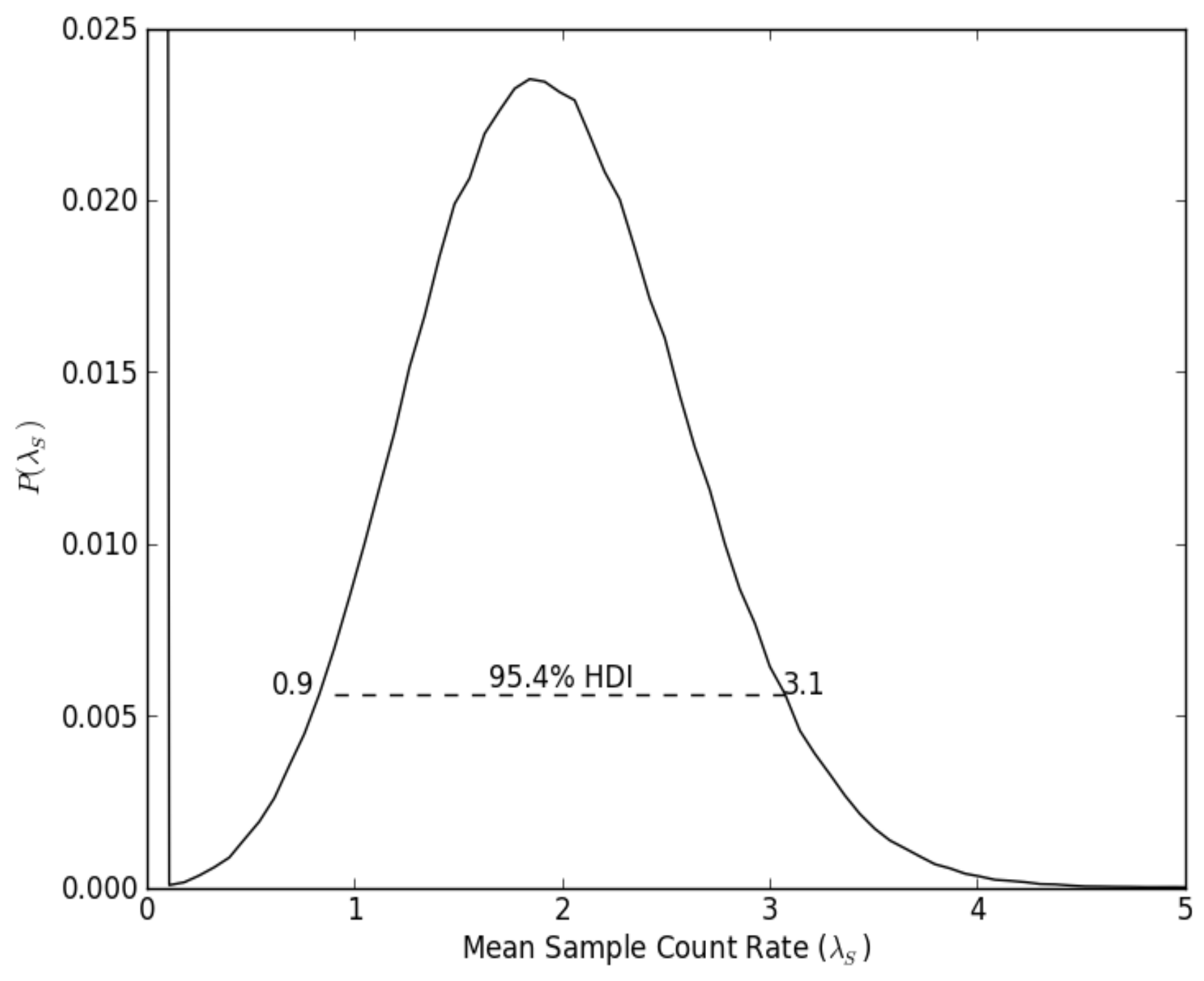




\section{Summary}

- The number of counts detected from a radioactive source is a random number sampled from an unknown probability distribution

- We try to figure out what that probability distribution is by doing measurements

- Whether or not a sample is radioactive, and the count rate of a radioactive source, are also random numbers sampled from some probability distribution

- To reach the right conclusions from data, we need to estimate some probabilities in advance (e.g., the probability that the source is radioactive) 


\section{Homework Questions}

- Suppose you flip a coin ten times. How likely is it that it will come up heads 8 times? Hint: This is the equation on slide 9 with $p=1 / 2$ and $n=10$. Is this enough to convince you that the coin is 'weighted' (i.e. not fair). What if you flipped it 20 times and it came up heads 18 times?

Suppose there is a pandemic. One in 1,000 people in your neighborhood have the disease at any given time. You are randomly selected for a test. If you have the disease, there is a $100 \%$ chance that the test will give you a positive result. However, if you do not have the disease, the test will issue a 'false-positive' $5 \%$ of the times. What are the chances you have the disease if you test positive? How would your odds change if the pandemic was worse, and one in 100 people had the disease at any given time? Hint: Following slide 15, imagine that 1,000 people took the test, and figure out how many sick and how many health people test positive.

- You measure a source that is supposed to have 20 decays per minute, but you measure 23 decays in one minute. Does this mean your detector is broken? Are you measuring the wrong source? Explain your answer.

- The half-life of plutonium-239 is 24,000 years. What is $\lambda$ ?

- You excrete about one millions plutonium atoms per day in your urine because of fallout from atmospheric nuclear weapons testing. What is the decay rate of one million atoms of plutonium-239?

- In Example 2, what is the probability that the source you have measured is radioactive? 